\title{
A NOTE ON REDUCTIONS OF 2-DIMENSIONAL CRYSTALLINE GALOIS REPRESENTATIONS
}

\author{
GERASIMOS DOUSMANIS
}

(Communicated by Matthew A. Papanikolas)

\begin{abstract}
Let $p$ be an odd prime number, $K_{f}$ the finite unramified extension of $\mathbb{Q}_{p}$ of degree $f$ and $G_{K_{f}}$ its absolute Galois group. We construct analytic families of étale $\left(\varphi, \Gamma_{K_{f}}\right)$-modules which give rise to some families of 2-dimensional crystalline representations of $G_{K_{f}}$ with length of filtration $\geq p$. As an application we prove that the modulo $p$ reductions of the members of each such family (with respect to appropriately chosen Galois-stable lattices) are constant.
\end{abstract}

\section{INTRODUCTION}

Two-dimensional crystalline representations of $G_{\mathbb{Q}_{p}}:=\mathrm{Gal}\left(\overline{\mathbb{Q}_{p}} / \mathbb{Q}_{p}\right)$ arise from classical newforms of level prime to $p$. Modulo $p$ reductions of such representations with Hodge-Tate weights in the range $[0 ; p]$ mattered for the proof of Serre's modularity conjecture by Khare and Wintenberger. The reductions of all irreducible 2-dimensional crystalline representations of $G_{\mathbb{Q}_{p}}$ with Hodge-Tate weights in this range were computed by Berger-Li-Zhu [4, extending previous results of Deligne, Edixhoven, Fontaine and Serre.

Serre's modularity conjecture was generalized by Buzzard, Diamond and Jarvis [5] for irreducible totally odd 2-dimensional $\overline{\mathbb{F}_{p}}$-representations of the absolute Galois group of any totally real field unramified at $p$. Two-dimensional crystalline representations of $G_{K_{f}}:=\mathrm{Gal}\left(\overline{\mathbb{Q}_{p}} / K_{f}\right)$ arise naturally in the context of the BDJ conjecture, and their modulo $p$ reductions are important for the formulation of the weight part of this conjecture (see [5, §3]). The weight part of Serre's conjecture for rank two unitary groups, for modulo $p$ representations in the unramified case, has recently been proved by Gee, Liu and Savitt [11, using Liu's theory of $(\varphi, \hat{G})$ modules developed in [12]. However, they have been unable to explicitly determine the modulo $p$ reduction of a given 2-dimensional crystalline $\overline{\mathbb{Q}_{p}}$-representation of $G_{K_{f}}$. Instead, they determined the possible modulo $p$ reductions in terms of the Hodge-Tate weights, when the length of the filtration of the corresponding admissible filtered $\varphi$-module is $\leq p$.

For arbitrary Hodge-Tate weights, semisimplified modulo $p$ reductions of certain families of 2-dimensional crystalline representations of $G_{K_{f}}$ were computed in [8], extending the constructions in [4] from $\mathbb{Q}_{p}$ to $K_{f}$. More precisely, for any 2dimensional crystalline representation $V$ of $G_{K_{f}}$ with Hodge-Tate type $\operatorname{HT}_{V}(\tau)=$ $\left\{0,-k_{i}\right\}$, where $k_{i}$ are non-negative integers, which is up to unramified twist either

Received by the editors April 18, 2012 and, in revised form, December 3, 2012.

2010 Mathematics Subject Classification. Primary 11F80, 11F85. 
irreducible and induced from a crystalline character of $G_{K_{2 f}}$ or split-reducible and non-ordinary, we constructed an infinite family $\mathcal{F}(V)$ of 2-dimensional crystalline representations of $G_{K_{f}}$ with the following properties:

(1) $V \in \mathcal{F}(V)$.

(2) The members of $\mathcal{F}(V)$ have Hodge-Tate type $\operatorname{HT}_{V}(\tau)$.

(3) The members of $\mathcal{F}(V)$ have the same modulo $p$ reductions with respect to appropriately chosen Galois-stable lattices.

The crystalline representations of $\mathcal{F}(V)$ were described in terms of their corresponding by the Colmez-Fontaine theorem (see [6, Théorèm A]) admissible filtered $\varphi$-modules. For each family $\mathcal{F}(V)$, the semisimplification $\overline{\mathcal{F}(V)}$ ss of the common reduction is independent of choices of lattices and was explicitly computed in ([8. Theorems 1.5 and 1.7]). Recall that if $V$ is reducible, $\mathcal{F}(V)$ can contain both irreducible and reducible representations (see 8, comments after Theorem 1.7).

The modulo $p$ reduction of a given 2-dimensional crystalline $\overline{\mathbb{Q}_{p}}$-representation of $G_{K_{f}}$ is generally unknown, even if the length of its filtration is $\leq p$, when $f \geq 2$. The goal of this paper is to enlarge the families $\mathcal{F}(V)$ to families of 2-dimensional crystalline $\overline{\mathbb{Q}_{p}}$-representations of the same Hodge-Tate type and constant modulo $p$ reductions with respect to appropriately chosen Galois-stable lattices, under the assumption that the length of filtration of $V$ is at least $p \neq 2$. In addition to their application to Serre's modularity conjecture the results of [4] provided evidence for the existence of the $p$-adic Langlands correspondence for $\mathbb{Q}_{p}$, and we expect that our results will similarly allow one to test the $p$-adic Langlands correspondence for $K_{f}$ currently being developed. The proof of our theorem rests on Wach module constructions and makes use of the constructions in [8] and an idea of Berger (3. $\S 10.3])$.

\section{Description of the FAMilies}

Throughout this paper $p$ will be a fixed odd integer prime, $K_{f}=\mathbb{Q}_{p^{f}}$ the finite unramified extension of $\mathbb{Q}_{p}$ of degree $f$, and $E$ a finite extension of $K_{f}$ with ring of integers $\mathcal{O}_{E}$, maximal ideal $\mathfrak{m}_{E}$, and residue field $k_{E}$. When the degree of $K_{f}$ plays no role we simply write $K$. We denote by $\sigma_{K}$ the absolute Frobenius of $K$; we fix once and for all a distinguished embedding $K \stackrel{\tau_{0}}{\hookrightarrow} E$ and we let $\tau_{j}=\tau_{0} \circ \sigma_{K}^{j}$ for all $j=0,1, \ldots, f-1$. We fix the $f$-tuple of embeddings $|\tau|:=\left(\tau_{0}, \tau_{1}, \ldots, \tau_{f-1}\right)$; we denote $E^{|\tau|}:=\prod_{\tau: K \hookrightarrow E} E$, with the embeddings ordered as above; and we let $e_{i}:=\left(0, \ldots, 0,1_{i}, 0, \ldots, 0\right) \in E^{|\tau|}$ for $i=0,1, \ldots, f-1$. For the language of crystalline representations see [9].

Notation 1.1. For $i=0,1, \ldots, f-1$, let $k_{i}$ be fixed non-negative integers which we call weights. Assume that after ordering them and omitting possibly repeated weights we get $w_{0}<w_{1}<\cdots<w_{t-1}$, where $w_{0}$ is the smallest weight, $w_{1}$ the second smallest weight, $\ldots$, and $w_{t-1}$ the largest weight for some $1 \leq t \leq f$. The largest weight $w_{t-1}$ will usually be denoted by $k$, and throughout the paper we assume that $k \geq p$. For convenience we define $w_{-1}=0$. Let $I_{0}:=\{0,1, \ldots, f-1\}$; for $j=1,2, \ldots, t-1$ we let $I_{j}:=\left\{i \in I_{0}: k_{i}>w_{j-1}\right\}$, and $I_{t}=\varnothing$. For each subset $J \subset I_{0}$ we write $f_{J}:=\sum_{i \in J} e_{i}$ and $E^{\left|\tau_{J}\right|}:=f_{J} \cdot E^{|\tau|}$. The sets $E^{\left|\tau_{I_{j}}\right|}$ are obtained as follows: $E^{\left|\tau_{I_{0}}\right|}$ is the Cartesian product $E^{f}$. Starting with $E^{\left|\tau_{I_{0}}\right|}$, we obtain $E^{\left|\tau_{I_{1}}\right|}$ 
by killing the coordinates where the smallest weight occurs. We obtain $E^{\left|\tau_{I_{2}}\right|}$ by further killing the coordinates where the second smallest weight $w_{1}$ occurs, and so on.

We first recall the construction of the families $\mathcal{F}(V)$ in $[8$. For $i=0,1, \ldots, f-1$, let $\chi_{i}$ be a crystalline $E$-character of $G_{K_{f}}$ with Hodge-Tate type $\operatorname{HT}_{\chi_{i}}\left(\tau_{i+1}\right)=$ $\{-1\}$ and $\mathrm{HT}_{\chi_{j}}\left(\tau_{i+1}\right)=\{0\}$ if $j \neq i+1$, where the indices are viewed modulo $f$. Let $\left\{\ell_{j}\right\}_{0 \leq j \leq 2 f-1}$ be integers such that $\left\{\ell_{i}, \ell_{f+i}\right\}=\left\{0, k_{i}\right\}$ for all $i=0,1, \ldots, f-1$. Up to unramified twist, any irreducible 2-dimensional crystalline representation $V$ of $G_{K_{f}}$ of Hodge-Tate type $\mathrm{HT}_{V}(\tau)=\left\{0,-k_{i}\right\}$ induced from a crystalline character of $G_{K_{2 f}}$ has the form $V=\operatorname{Ind}_{K_{2 f}}^{K_{f}}\left(\chi_{\vec{\ell}}\right)$, where $\chi_{\vec{\ell}}=\chi_{0}^{\ell_{1}} \cdot \chi_{1}^{\ell_{2}} \cdots \chi_{2 f-2}^{\ell_{2 f-1}} \cdot \chi_{2 f-1}^{\ell_{0}}$ (cf. [8, Theorem 1.3]). Any split-reducible non-ordinary 2-dimensional crystalline representation $V$ of $G_{K_{f}}$ of Hodge-Tate type $\operatorname{HT}_{V}(\tau)=\left\{0,-k_{i}\right\}$ is up to unramified twist of the form

$$
V=\eta \cdot \chi_{0}^{\ell_{1}} \cdot \chi_{1}^{\ell_{2}} \cdots \chi_{f-2}^{\ell_{f-1}} \cdot \chi_{f-1}^{\ell_{0}} \oplus \chi_{0}^{\ell_{1+f}} \cdot \chi_{1}^{\ell_{2+f}} \cdots \chi_{f-2}^{\ell_{2 f-1}} \cdot \chi_{f-1}^{\ell_{f}},
$$

where $\eta$ is an unramified character, with both vectors $\left(\ell_{0}, \ell_{1}, \ldots, \ell_{f-1}\right)$ and $\left(\ell_{f}, \ell_{f+1}\right.$, $\left.\ldots, \ell_{2 f-1}\right)$ non-zero (cf. [8, Theorem 1.7]). Fix a representation $V$ as above. Let $\left\{X_{i}\right\}_{1 \leq i \leq f}$ be a set of indeterminates and let $P_{i}\left(X_{i}\right) \in M_{2}\left(\mathcal{O}_{E}\left[X_{i}\right]\right)$ be a matrix of one of the following four types:

$$
t_{1}:\left(\begin{array}{cc}
p^{k_{i}} & 0 \\
X_{i} & 1
\end{array}\right), \quad t_{2}:\left(\begin{array}{cc}
X_{i} & 1 \\
p^{k_{i}} & 0
\end{array}\right), \quad t_{3}:\left(\begin{array}{cc}
1 & X_{i} \\
0 & p^{k_{i}}
\end{array}\right), \quad t_{4}:\left(\begin{array}{cc}
0 & p^{k_{i}} \\
1 & X_{i}
\end{array}\right) .
$$

Let $P(\vec{X})=\left(P_{1}\left(X_{1}\right), P_{2}\left(X_{2}\right), \ldots, P_{f}\left(X_{f}\right)\right)$, where the indices are viewed $\bmod f$, and choose the type of the matrix $P_{i}\left(X_{i}\right)$ as follows: If $f=1$ we choose $P_{1}=t_{2}$. Assume that $f \geq 2$.

Case (i). $V$ is induced.

(1) If $\ell_{1}=0, P_{1}=t_{2}$.

(2) If $\ell_{1}=k_{1}>0, P_{1}=t_{1}$.

For $i=2,3, \ldots, f-1$ we choose the type of the matrix $P_{i}$ as follows:

(1) If $\ell_{i}=0$, then:

- If an even number of coordinates of $\left(P_{1}, P_{2}, \ldots, P_{i-1}\right)$ is of even type, $P_{i}=$ $t_{2}$.

- If an odd number of coordinates of $\left(P_{1}, P_{2}, \ldots, P_{i-1}\right)$ is of even type, $P_{i}=$ $t_{1}$.

(2) If $\ell_{i}=k_{i}>0$, then:

- If an even number of coordinates of $\left(P_{1}, P_{2}, \ldots, P_{i-1}\right)$ is of even type, $P_{i}=$ $t_{1}$.

- If an odd number of coordinates of $\left(P_{1}, P_{2}, \ldots, P_{i-1}\right)$ is of even type, $P_{i}=$ $t_{2}$.

Finally, we choose the type of the matrix $P_{0}:=P_{f}$ as follows:

(1) If $\ell_{0}=0$, then:

- If an even number of coordinates of $\left(P_{1}, P_{2}, \ldots, P_{f-1}\right)$ is of even type, $P_{0}=$ $t_{4}$.

- If an odd number of coordinates of $\left(P_{1}, P_{2}, \ldots, P_{f-1}\right)$ is of even type, $P_{0}=$ $t_{3}$. 
(2) If $\ell_{0}=k_{0}>0$, then:

- If an even number of coordinates of $\left(P_{1}, P_{2}, \ldots, P_{f-1}\right)$ is of even type, $P_{0}=$ $t_{2}$.

- If an odd number of coordinates of $\left(P_{1}, P_{2}, \ldots, P_{f-1}\right)$ is of even type, $P_{0}=$ $t_{1}$.

Case (ii). $V$ is split reducible and non-ordinary.

The $(f-1)$-tuple $\left(P_{1}, P_{2}, \ldots, P_{f-1}\right)$ is chosen as in Case (i) above. If $\eta=\eta_{c}$ is the unramified character which maps the geometric Frobenius element Frob $K_{f}$ of $G_{K_{f}}$ to $c$, we replace the entry $p^{k_{0}}$ in the definition of the matrix $P_{0}$ by $c p^{k_{0}}$. The type of the matrix $P_{0}:=P_{f}$ is chosen as follows:

(1) If $\ell_{0}=0$, then:

- If an even number of coordinates of $\left(P_{1}, P_{2}, \ldots, P_{f-1}\right)$ is of even type, $P_{0}=$ $t_{3}$.

- If an odd number of coordinates of $\left(P_{1}, P_{2}, \ldots, P_{f-1}\right)$ is of even type, $P_{0}=$ $t_{4}$.

(2) If $\ell_{0}=k_{0}>0$, then:

- If an even number of coordinates of $\left(P_{1}, P_{2}, \ldots, P_{f-1}\right)$ is of even type, $P_{0}=$ $t_{1}$.

- If an odd number of coordinates of $\left(P_{1}, P_{2}, \ldots, P_{f-1}\right)$ is of even type, $P_{0}=$ $t_{2}$.

Recall that $k \geq p$ and let

$$
m:=\left\{\begin{array}{cl}
\left\lfloor\frac{k-1}{p-1}\right\rfloor & \text { if } k_{i} \neq p \text { for some } i, \\
0 & \text { if } k_{i}=p \text { for all } i .
\end{array}\right.
$$

For any $\vec{\alpha}=\left(\alpha_{1}, \alpha_{2}, \ldots, \alpha_{f}\right) \in\left(p^{m} \mathfrak{m}_{E}\right)^{f}$, let $P(\vec{\alpha})$ be the matrix obtained by evaluating each indeterminate $X_{i}$ at $\alpha_{i}$. We defined $\mathcal{F}(V)$ as the family of 2dimensional crystalline representations $\left\{V(\vec{\alpha}), \vec{\alpha} \in\left(p^{m} \mathfrak{m}_{E}\right)^{f}\right\}$ corresponding by the Colmez-Fontaine theorem to the family of admissible filtered $\varphi$-modules obtained by equipping $\mathbb{D}(\vec{\alpha})=E^{|\tau|} \eta_{1} \oplus E^{|\tau|} \eta_{2}$ with the Frobenius action defined by $\left(\varphi\left(\eta_{1}\right), \varphi\left(\eta_{2}\right)\right)=\left(\eta_{1}, \eta_{2}\right) P(\vec{\alpha})$ and the filtration

$\operatorname{Fil}^{\mathrm{j}}(\mathbb{D}(\vec{\alpha}))=\left\{\begin{array}{cl}E^{|\tau|} \eta_{1} \oplus E^{|\tau|} \eta_{2} & \text { if } j \leq 0, \\ E^{\left|\tau_{I_{s}}\right|}\left(\vec{x} \eta_{1}+\vec{y} \eta_{2}\right) & \text { if } 1+w_{s-1} \leq j \leq w_{s}, \text { for } s=0,1, \ldots, t-1, \\ 0 & \text { if } j \geq 1+w_{t-1},\end{array}\right.$

where $\vec{x}=\left(x_{0}, x_{1}, \ldots, x_{f-1}\right)$ and $\vec{y}=\left(y_{0}, y_{1}, \ldots, y_{f-1}\right)$, with

$$
\left(x_{i}, y_{i}\right)= \begin{cases}\left(1,-\alpha_{i}\right) & \text { if } P_{i} \text { has type } 1 \text { or } 2, \\ \left(-\alpha_{i}, 1\right) & \text { if } P_{i} \text { has type } 3 \text { or } 4,\end{cases}
$$

for any $\vec{\alpha} \in\left(p^{m} \mathfrak{m}_{E}\right)^{f}$. By the construction of these families in [8] it follows that $V(\overrightarrow{0})=V$. We now enlarge each such family $\mathcal{F}(V)$, preserving the Hodge-Tate types and leaving unchanged the modulo $p$ reductions with respect to appropriately chosen Galois-stable $\mathcal{O}_{E}$-lattices.

Let $\alpha(k):=\sum_{n=0}^{\infty}\left\lfloor\frac{k}{p^{n}(p-1)}\right\rfloor$. For any $A=\left(A_{1}, A, \ldots, A_{f}\right) \in M_{2}\left(p^{\alpha(k-1)} \mathcal{O}_{E}\right)^{|\tau|}$ we define

$$
P_{A}(\vec{X}):=(\overrightarrow{I d}+A) P(\vec{X}) .
$$


If $\vec{\alpha} \in\left(p^{m} \mathfrak{m}_{E}\right)^{f}$ and $A \in M_{2}\left(p^{\alpha(k-1)} \mathcal{O}_{E}\right)^{|\tau|}$, we denote by $\left(\mathbb{D}_{A}(\vec{\alpha}), \varphi\right)$ the filtered $\varphi$-module obtained by equipping $\mathbb{D}_{A}(\vec{\alpha})=E^{|\tau|} \eta_{1} \oplus E^{|\tau|} \eta_{2}$ with the Frobenius endomorphism defined by $\left(\varphi\left(\eta_{1}\right), \varphi\left(\eta_{2}\right)\right)=\left(\eta_{1}, \eta_{2}\right) P_{A}(\vec{\alpha})$ and with the same filtration as $(\mathbb{D}(\vec{\alpha}), \varphi)$ independently of $A$. Such a filtered $\varphi$-module turns out to be admissible. Let $V_{A}(\vec{\alpha})$ be the crystalline representation corresponding by the Colmez-Fontaine theorem to $\left(\mathbb{D}_{A}(\vec{\alpha}), \varphi\right)$, and let

$\mathcal{G}(V)=\bigcup_{A \in \mathcal{M}(k)}\left\{V_{A}(\vec{\alpha}): \vec{\alpha} \in\left(p^{m} \mathfrak{m}_{E}\right)^{f}\right\}$, where $\mathcal{M}(k)=M_{2}\left(p^{1+\alpha(k-1)} \mathcal{O}_{E}\right)^{|\tau|}$.

Theorem A. (i) For any $\vec{\alpha} \in\left(p^{m} \mathfrak{m}_{E}\right)^{f}$ and any $A \in M_{2}\left(p^{\alpha(k-1)} \mathcal{O}_{E}\right)^{|\tau|}$, the filtered $\varphi$-modules $\mathbb{D}_{A}(\vec{\alpha})$ are admissible and the corresponding crystalline representations have Hodge-Tate type $\operatorname{HT}\left(\tau_{i}\right)=\left\{0,-k_{i}\right\}$.

(ii) For any $\vec{\alpha} \in\left(p^{m} \mathfrak{m}_{E}\right)^{f}$ and any $A \in M_{2}\left(p^{\alpha(k-1)} \mathcal{O}_{E}\right)^{|\tau|}$, there exist $G_{K_{f}}$ stable $\mathcal{O}_{E}$-lattices with respect to which $\bar{V}_{A}(\vec{\alpha})=\bar{V}_{A}(\overrightarrow{0})$.

(iii) There exist $G_{K_{f}}$-stable $\mathcal{O}_{E}$-lattices with respect to which all members of $\mathcal{G}(V)$ have the same modulo $p$ reduction $\overline{\mathcal{G}(V)}$. Moreover, $\overline{\mathcal{G}(V)}=\bar{V}$.

Remark 1.2. (1) By ([즈, Theorems 1.5 and 1.7]),

$$
\left({\overline{\mathcal{G}}(V)_{\mid I_{K_{f}}}}^{s s}=\left\{\begin{array}{l}
\omega_{2 f, \bar{\tau}_{0}}^{\beta} \oplus \omega_{2 f, \bar{\tau}_{0}}^{p^{f} \beta}, \text { where } \beta=-\sum_{i=0}^{2 f-1} \ell_{i} p^{i} \text { if } V \text { is irreducible and induced, } \\
\omega_{f, \bar{\tau}_{0}}^{\beta} \oplus \omega_{f, \bar{\tau}_{0}}^{\beta^{\prime}}, \text { where } \beta=-\sum_{i=0}^{f-1} \ell_{i} p^{i} \text { and } \beta^{\prime}=-\sum_{i=0}^{f-1} \ell_{i+f} p^{i} \text { if } V \text { is } \\
\text { split-reducible and non-ordinary. }
\end{array}\right.\right.
$$

Recall that the level $f$ fundamental character $\omega_{f, \bar{\tau}_{0}}: I_{K_{f}} \rightarrow k_{E}^{\times}$is obtained by composing the homomorphism $I_{K_{f}} \rightarrow k_{K_{f}}^{\times}$obtained from local class field theory (with uniformizers corresponding to geometric Frobenius elements) with the embedding of residue fields $k_{K_{f}} \stackrel{\bar{\tau}_{0}}{\rightarrow} k_{E}$ obtained from the distinguished embedding $K \stackrel{\tau_{0}}{\hookrightarrow} E$.

(2) For the rest of this remark assume that $f \geq 2$. In this case, for a 2-dimensional crystalline $\overline{\mathbb{Q}_{p}}$-representation $V$ of $G_{K_{f}}$, the characteristic polynomial of Frobenius and a choice of filtration fail to determine its isomorphism class. Assuming that $V$ is Frobenius-semisimple and non-Frobenius-scalar and fixing the characteristic polynomial of Frobenius and a choice for the filtration, the additional datum required to determine the isomorphism class of $V$ is (roughly) an element of $\mathbb{P}^{f-1}(E)$ (for a precise statement see [7, $\left.\S 7\right]$ ). The isomorphism classes of non-Frobenius-semisimple or Frobenius-scalar filtered $\varphi$-modules are in general messier to describe (see [7, $\S 6]$ ).

(3) The representations of $\mathcal{G}(V)$ yield additional "projective parameters" compared to the set of "projective parameters" attached to the Frobeniussemisimple and non-Frobenius-scalar unramified twists of members of $\mathcal{F}(V)$. However, they yield no new characteristic polynomials or filtrations.

(4) The formulas for the "projective parameters" of the Frobenius-semisimple and non-Frobenius-scalar representations of the families $\mathcal{G}(V)$ look particularly abhorrent (see for instance the proof of [8, Proposition 6.21]). The situation becomes even worse with the non-Frobenius-semisimple, and especially with the Frobenius-scalar, members of these families. This makes it hard to give a clean description, in terms of the classification of admissible 
filtered $\varphi$-modules obtained in [7, of how many 2-dimensional crystalline representations of $G_{K_{f}}$ with Hodge-Tate weights in the range $[0 ; p]$ we are able to compute the semisimplified modulo $p$ reduction of, using Theorem $\mathrm{A}$, and what is possibly missing.

(5) Theorem A can be thought of as a local constancy result for modulo $p$ reductions of 2-dimensional crystalline representations of $G_{K_{f}}$ within certain families. For results of a similar flavor for 2-dimensional crystalline representations of $G_{\mathbb{Q}_{p}}$, see 2 .

\section{FAmilies of Wach MOdules}

2.1. Étale $(\varphi, \Gamma)$-modules and Wach modules. Let $\mathcal{K}_{n}=K\left(\zeta_{p^{n}}\right)$, where $\zeta_{p^{n}}$ is a primitive $p^{n}$-th root of unity inside $\overline{\mathbb{Q}_{p}}$, and let $K_{\infty}=\bigcup_{n \geq 1} \mathcal{K}_{n}$. Let $\chi: G_{K} \rightarrow \mathbb{Z}_{p}^{\times}$ be the cyclotomic character, $H_{K}:=\operatorname{ker} \chi=\operatorname{Gal}\left(\overline{\mathbb{Q}_{p}} / K_{\infty}\right)$, and $\Gamma_{K}:=G_{K} / H_{K}=$ $\operatorname{Gal}\left(K_{\infty} / K\right)$. Fontaine [10] has constructed topological rings $\mathbb{A}$ and $\mathbb{B}$ endowed with continuous commuting Frobenius $\varphi$ and $G_{\mathbb{Q}_{p}}$-actions. Unless otherwise stated and whenever applicable, continuity will mean continuity with respect to the topologies induced by the weak topologies of the topological rings $\mathbb{A}$ and $\mathbb{B}$. Let $\mathbb{A}_{K}=\mathbb{A}^{H_{K}}$ and $\mathbb{B}_{K}=\mathbb{B}^{H_{K}}$, and let $\mathbb{A}_{K, E}:=\mathcal{O}_{E} \otimes_{\mathbb{Z}_{p}} \mathbb{A}_{K}$ and $\mathbb{B}_{K, E}:=E \otimes_{\mathbb{Q}_{p}} \mathbb{B}_{K}$. The actions of $\varphi$ and $\Gamma_{K}$ extend to $\mathbb{A}_{K, E}$ and $\mathbb{B}_{K, E}$ by $\mathcal{O}_{E}$ (resp. E)-linearity, and one easily sees that $\mathbb{A}_{K, E}=\mathbb{A}_{E}^{H_{K}}$ and $\mathbb{B}_{K, E}=\mathbb{B}_{E}^{H_{K}}$.

Definition 2.1. A $(\varphi, \Gamma)$-module over $\mathbb{A}_{K, E}$ (resp. $\left.\mathbb{B}_{K, E}\right)$ is an $\mathbb{A}_{K, E}$-module of finite type (resp. a free $\mathbb{B}_{K, E}$-module of finite type) endowed with a semilinear and continuous action of $\Gamma_{K}$, and with a semilinear map $\varphi$ which commutes with the action of $\Gamma_{K}$. A $(\varphi, \Gamma)$-module $M$ over $\mathbb{A}_{K, E}$ is called étale if $\varphi^{*}(M)=M$, where $\varphi^{*}(M)$ is the $\mathbb{A}_{K, E}$-module generated by the set $\varphi(M)$. A $(\varphi, \Gamma)$-module $M$ over $\mathbb{B}_{K, E}$ is called étale if it contains a basis $\left(e_{1}, \ldots, e_{d}\right)$ over $\mathbb{B}_{K, E}$ such that $\left(\varphi\left(e_{1}\right), \ldots, \varphi\left(e_{d}\right)\right)=\left(e_{1}, \ldots, e_{d}\right) A$, for some matrix $A \in \mathrm{GL}_{d}\left(\mathbb{A}_{K, E}\right)$.

If $V$ is a continuous $E$-linear representation of $G_{K}$ we equip the $\mathbb{B}_{K, E}$-module $\mathbb{D}(V):=\left(\mathbb{B}_{E} \otimes_{E} V\right)^{H_{K}}$ with a Frobenius endomorphism $\varphi$ defined by $\varphi(b \otimes v):=$ $\varphi(b) \otimes v$, where $\varphi$ on the right hand side is the Frobenius of $\mathbb{B}_{E}$, and with an action of $\Gamma_{K}$ given by $\bar{g}(b \otimes v):=g b \otimes g v$ for any $g \in G_{K}$. This $\Gamma_{K}$-action commutes with $\varphi$ and is continuous. Moreover, $\mathbb{D}(V)$ is an étale $(\varphi, \Gamma)$-module over $\mathbb{B}_{K, E}$. Conversely, if $D$ is an étale $(\varphi, \Gamma)$-module over $\mathbb{B}_{K, E}$, let $\mathbb{V}(D):=\left(\mathbb{B}_{E} \otimes_{\mathbb{B}_{K, E}} D\right)^{\varphi=1}$, where $\varphi(b \otimes d):=\varphi(b) \otimes \varphi(d)$. The $E$-vector space $\mathbb{V}(D)$ is finite dimensional and is equipped with a continuous $E$-linear $G_{K}$-action given by $g(b \otimes d):=g b \otimes \bar{g} d$. We have the following theorem of Fontaine.

Theorem 2.2 ([10]).

(i) There is an equivalence of categories between continuous E-linear representations of $G_{K}$ and étale $(\varphi, \Gamma)$-modules over $\mathbb{B}_{K, E}$ given by

$\mathbb{D}: \operatorname{Rep}_{E}\left(G_{K}\right) \rightarrow \mathcal{M o d}_{(\varphi, \Gamma)}^{e ́ t}\left(\mathbb{B}_{K, E}\right): V \longmapsto \mathbb{D}(V):=\left(\mathbb{B}_{E} \otimes_{E} V\right)^{H_{K}}$, with quasi-inverse functor

$$
\mathbb{V}: \operatorname{Mod}_{(\varphi, \Gamma)}^{\text {ét }}\left(\mathbb{B}_{K, E}\right) \rightarrow \operatorname{Rep}_{E}\left(G_{K}\right): D \longmapsto \mathbb{V}(D):=\left(\mathbb{B}_{E} \otimes_{\mathbb{B}_{K, E}} D\right)^{\varphi=1}
$$


(ii) There is an equivalence of categories between continuous $\mathcal{O}_{E}$-linear representations of $G_{K}$ and étale $(\varphi, \Gamma)$-modules over $\mathbb{A}_{K, E}$ given by

$\mathbb{D}: \operatorname{Rep}_{\mathcal{O}_{E}}\left(G_{K}\right) \rightarrow \operatorname{Mod}_{(\varphi, \Gamma)}{ }_{(e ́ t}\left(\mathbb{A}_{K, E}\right): \mathrm{T} \longmapsto \mathbb{D}(T):=\left(\mathbb{A}_{E} \otimes_{\mathcal{O}_{E}} \mathrm{~T}\right)^{H_{K}}$, with quasi-inverse functor

$$
\mathbb{T}: \operatorname{Mod}_{(\varphi, \Gamma)}^{e ́ t}\left(\mathbb{A}_{K, E}\right) \rightarrow \operatorname{Rep}_{\mathcal{O}_{E}}\left(G_{K}\right): D \longmapsto \mathbb{T}(D):=\left(\mathbb{A}_{E} \otimes_{\mathbb{A}_{K, E}} D\right)^{\varphi=1} .
$$

Let $\mathbb{A}_{K}=\left\{\sum_{-\infty}^{+\infty} \alpha_{n} \pi^{n}: \alpha_{n} \in \mathcal{O}_{K}\right.$ and $\left.\lim _{n \rightarrow-\infty} \alpha_{n}=0\right\}$ for some element $\pi$ which can be thought of as a formal variable. The ring $\mathbb{A}_{K}$ is equipped with a Frobenius endomorphism $\varphi$ which extends the absolute Frobenius of $\mathcal{O}_{K}$ and is such that $\varphi(\pi)=(1+\pi)^{p}-1$. It is also equipped with a $\Gamma_{K}$-action which is $\mathcal{O}_{K}$-linear, commutes with Frobenius, and is such that $\gamma(\pi)=(1+\pi)^{\chi(\gamma)}-1$ for all $\gamma \in \Gamma_{K}$. The ring $\mathbb{A}_{K}$ is a local domain with maximal ideal $(p)$ and fraction field $\mathbb{B}_{K}=\mathbb{A}_{K}\left[\frac{1}{p}\right]$. The rings $\mathbb{A}_{K}, \mathbb{A}_{K, E}, \mathbb{B}_{K}$ and $\mathbb{B}_{K, E}$ contain the subrings $\mathbb{A}_{K}^{+}=\mathcal{O}_{K}[[\pi]], \mathbb{A}_{K, E}^{+}:=$ $\mathcal{O}_{E} \otimes_{\mathbb{Z}_{p}} \mathbb{A}_{K}^{+}, \mathbb{B}_{K}^{+}=\mathbb{A}_{K}^{+}\left[\frac{1}{p}\right]$ and $\mathbb{B}_{K, E}^{+}:=E \otimes_{\mathbb{Q}_{p}} \mathbb{B}_{K}^{+}$respectively, and these subrings are equipped with the restrictions of the $\varphi$ and the $\Gamma_{K}$-actions of the rings containing them. The map $v: \mathbb{A}_{K, E}^{+} \rightarrow \prod_{\tau: K \hookrightarrow E} \mathcal{O}_{E}[[\pi]]$ given by $v(a \otimes b)=$ $\left(a \tau_{0}(b), a \tau_{1}(b), \ldots, a \tau_{f-1}(b)\right)$, where $\tau_{i}\left(\sum_{n=0}^{\infty} \beta_{n} \pi^{n}\right)=\sum_{n=0}^{\infty} \tau_{i}\left(\beta_{n}\right) \pi^{n}$ for all $b=\sum_{n=0}^{\infty} \beta_{n} \pi^{n} \in \mathbb{A}_{K}^{+}$, is a ring isomorphism. The ring $\mathcal{O}_{E}[[\pi]]^{|\tau|}$ $:=\prod_{\tau: K \hookrightarrow E} \mathcal{O}_{E}[[\pi]]$ is equipped via $v$ with commuting $\mathcal{O}_{E}$-linear actions of $\varphi$ and $\Gamma_{K}$ given by the formulas

$$
\begin{aligned}
& \varphi\left(\alpha_{0}(\pi), \alpha_{1}(\pi), \ldots, \alpha_{f-1}(\pi)\right)=\left(\alpha_{1}(\varphi(\pi)), \ldots, \alpha_{f-1}(\varphi(\pi)), \alpha_{0}(\varphi(\pi))\right) \text { and } \\
& \gamma\left(\alpha_{0}(\pi), \alpha_{1}(\pi), \ldots, \alpha_{f-1}(\pi)\right)=\left(\alpha_{0}(\gamma \pi), \alpha_{1}(\gamma \pi), \ldots, \alpha_{f-1}(\gamma \pi)\right)
\end{aligned}
$$

for all $\gamma \in \Gamma_{K}$.

Definition 2.3. Suppose $k \geq 0$. A Wach module over $\mathbb{A}_{K, E}^{+}\left(\right.$resp. $\left.\mathbb{B}_{K, E}^{+}\right)$with weights in $[-k ; 0]$ is a free $\mathbb{A}_{K, E}^{+}$-module (resp. $\mathbb{B}_{\left.K, E^{-m o d u l e}\right)}^{+} N$ of finite rank, endowed with an action of $\Gamma_{K}$ which becomes trivial modulo $\pi$, and also with a Frobenius map $\varphi$ which commutes with the action of $\Gamma_{K}$ and such that $\varphi(N) \subset N$ and $N / \varphi^{*}(N)$ is killed by $q^{k}$, where $q:=\varphi(\pi) / \pi$ and $\varphi^{*}(N)$ is the $\mathbb{A}_{K, E}^{+}$-module (resp. $\mathbb{B}_{K, E^{-}}^{+}$module) generated by the set $\varphi(N)$.

The following theorem of Berger determines which types of étale $(\varphi, \Gamma)$-modules correspond to crystalline representations via Fontaine's functor.

Theorem 2.4 ([1]).

(i) An E-linear representation $V$ of $G_{K}$ is crystalline with Hodge-Tate weights in $[-k ; 0]$ if and only if $\mathbb{D}(V)$ contains a unique Wach module $\mathbb{N}(V)$ of rank $\operatorname{dim}_{E} V$ with weights in $[-k ; 0]$. The functor $V \mapsto \mathbb{N}(V)$ defines an equivalence of categories between crystalline representations of $G_{K}$ and Wach modules over $\mathbb{B}_{K, E}^{+}$, compatible with tensor products, duality and exact sequences.

(ii) For a given crystalline E-representation $V$, the map $\mathrm{T} \mapsto \mathbb{N}(\mathrm{T}):=\mathbb{N}(V) \cap$ $\mathbb{D}(\mathrm{T})$ induces a bijection between $G_{K}$-stable, $\mathcal{O}_{E}$-lattices of $V$ and Wach modules over $\mathbb{A}_{K, E}^{+}$which are $\mathbb{A}_{K, E}^{+}$-lattices contained in $\mathbb{N}(V)$. Moreover, $\mathbb{D}(\mathrm{T})=\mathbb{A}_{K, E} \otimes_{\mathbb{A}_{K, E}^{+}} \mathbb{N}(\mathrm{T})$. 
(iii) If $V$ is a crystalline E-representation of $G_{K}$, and if we endow $\mathbb{N}(V)$ with the filtration $\mathrm{Fil}^{\mathrm{j}} \mathbb{N}(V)=\left\{x \in \mathbb{N}(V) \mid \varphi(x) \in q^{j} \mathbb{N}(V)\right\}$, then we have an isomorphism

$$
\mathbb{D}_{\text {cris }}(V) \rightarrow E^{|\tau|} \otimes_{\mathcal{O}_{E}} \mathbb{N}(V) / \pi \mathbb{N}(V)
$$

of filtered $\varphi$-modules over $E^{|\tau|}$ (with the induced filtration on $\mathbb{N}(V) / \pi \mathbb{N}(V)$ ).

2.2. Construction of families of Wach modules. We fix a topological generator $\delta$ of the procyclic group $\Gamma_{K}$. For any positive integer $\ell$, let

$$
\alpha(\ell):=\sum_{j=1}^{\ell} \mathrm{v}_{\mathrm{p}}(1-\chi(\delta))^{j}
$$

and let $\alpha(0)=0$. Recall that $\alpha(\ell)=0$ for $\ell \leq p-2$, while for an arbitrary $\ell, \alpha(\ell)=$ $\sum_{n=0}^{\infty}\left\lfloor\frac{\ell}{p^{n}(p-1)}\right\rfloor \leq\left\lfloor\frac{\ell p}{(p-1)^{2}}\right\rfloor$ (cf. [1, $\S$ IV.1]). Let $\mathcal{S}$ be a set of indeterminates and let $\pi$ be a distinguished indeterminate not belonging to $\mathcal{S}$. We denote by $M_{n}^{\mathcal{S}}$ the matrix $\operatorname{ring} M_{n}\left(\mathcal{O}_{E}[[\pi, \mathcal{S}]]\right)^{|\tau|}$. Recall that $k:=\max \left\{k_{i}\right\} \geq p$. For any integer $s \geq 0$ we write $\vec{\pi}^{s}=\left(\pi^{s}, \pi^{s}, \ldots, \pi^{s}\right)$, and we denote by $\overrightarrow{I d}$ the matrix of $M_{n}^{\mathcal{S}}$ whose coordinates are the identity matrix. We need the following variant of [3, Lemma 10.3.2].

Lemma 2.5. For each $\gamma \in \Gamma_{K}$, let $G_{\gamma}=G_{\gamma}(\mathcal{S}) \in \overrightarrow{I d}+\vec{\pi} M_{n}^{\mathcal{S}}$. Let $c \geq 0$ be an integer and let $A=\left(A_{1}, A_{2}, \ldots, A_{f}\right)$ be any matrix in $M_{n}\left(p^{c+\alpha(k-1)} \mathcal{O}_{E}\right)^{|\tau|}$. There exists a matrix

$$
\hat{A}=\left(\hat{A}_{1}, \hat{A}_{2}, \ldots, \hat{A}_{f}\right) \in M_{n}\left(p^{c} \mathcal{O}_{E}[[\pi, \mathcal{S}]]\right)^{|\tau|}
$$

such that:

(i) $\hat{A} \equiv A \bmod \vec{\pi}$.

(ii) $\overrightarrow{I d}+\hat{A} \in \mathrm{GL}_{n}\left(\mathcal{O}_{E}[[\pi, \mathcal{S}]]\right)^{|\tau|}$.

(iii) $(\overrightarrow{I d}+\hat{A}) \cdot G_{\gamma} \cdot \gamma(\overrightarrow{I d}+\hat{A})^{-1} \equiv G_{\gamma} \bmod \vec{\pi}^{k}$.

(iv) If $A \in M_{n}\left(p^{1+\alpha(k-1)} \mathcal{O}_{E}\right)^{|\tau|}$, then $\hat{A} \equiv 0 \bmod p$ and

$$
G_{\gamma}-(\overrightarrow{I d}+\hat{A}) \cdot G_{\gamma} \cdot \gamma(\overrightarrow{I d}+\hat{A})^{-1} \equiv 0 \bmod p
$$

Proof. Since $k \geq p$, for any $A \in M_{n}\left(p^{c+\alpha(k-1)} \mathcal{O}_{E}\right)^{|\tau|}$ it follows that $\overrightarrow{I d}+A \in$ $\mathrm{GL}_{n}\left(\mathcal{O}_{E}\right)^{|\tau|}$. Let $\hat{A}_{i}=A_{i}+\pi \hat{A}_{i}^{1}+\pi^{2} \hat{A}_{i}^{2}+\cdots+\pi^{k-1} \hat{A}_{i}^{k-1}$ and $G_{\gamma}=\left(G_{\gamma}^{1}, G_{\gamma}^{2}, \ldots, G_{\gamma}^{f}\right)$, with $G_{\gamma}^{i}=I d+\pi G_{1}^{i}+\pi^{2} G_{2}^{i}+\cdots+\pi^{k-1} G_{k-1}^{i}+\cdots$ (suppressing the dependence of $G_{j}^{i}$ on $\left.\gamma\right)$. We first show that $\overrightarrow{I d}+\hat{A} \in \mathrm{GL}_{n}\left(\mathcal{O}_{E}[[\pi, \mathcal{S}]]\right)^{|\tau|}$. Since $I d+A_{i} \in$ $I_{n}+M_{n}\left(p^{c+\alpha(k-1)} \mathcal{O}_{E}\right)$ for all $i$, each coordinate matrix $I d+\hat{A}_{i}$ is invertible and the inverse is $\left(I d+\hat{A}_{i}\right)^{-1}=\left(\sum_{n=0}^{\infty}(-1)^{n} \pi^{n} B_{i}^{n}\right)\left(I d+A_{i}\right)^{-1}$, where $B_{i}=$ $\left(I d+A_{i}\right)^{-1}\left(\hat{A}_{i}^{1}+\pi \hat{A}_{i}^{2}+\cdots+\pi^{k-2} \hat{A}_{i}^{k-1}\right)$. To prove part (iii), we need to choose the matrices $\hat{A}_{i}^{j}$ so that

$$
\begin{aligned}
(I d+ & \left.\pi G_{1}^{i}+\pi^{2} G_{2}^{i}+\cdots+\pi^{k-1} G_{k-1}^{i}+\cdots\right) \gamma\left(A_{i}+\pi \hat{A}_{i}^{1}+\pi^{2} \hat{A}_{i}^{2}+\cdots+\pi^{k-1} \hat{A}_{i}^{k-1}\right) \\
= & \left(A_{i}+\pi \hat{A}_{i}^{1}+\pi^{2} \hat{A}_{i}^{2}+\cdots+\pi^{k-1} \hat{A}_{i}^{k-1}\right) \\
& \cdot\left(I d+\pi G_{1}^{i}+\pi^{2} G_{2}^{i}+\cdots+\pi^{k-1} G_{k-1}^{i}+\cdots\right) \bmod \pi^{k} .
\end{aligned}
$$


We may assume that $\gamma$ is a topological generator of $\Gamma_{K}$. We solve for $\hat{A}_{i}^{j}$, bearing in mind that $\gamma(\pi)^{r} \equiv \chi(\gamma)^{r} \pi^{r} \bmod \pi^{r+1}$ for all $r \geq 1$. First, we solve for $\hat{A}_{i}^{1} \in M_{n}\left(\mathcal{O}_{E}[[\mathcal{S}]]\right)$ so that $(1-\chi(\gamma)) \hat{A}_{i}^{1}=A_{i} G_{1}^{i}-G_{1}^{i} A_{i}$. Since $A_{i} G_{1}^{i}-G_{1}^{i} A_{i} \in$ $M_{n}\left(p^{c+\alpha(k-1)} \mathcal{O}_{E}[[\mathcal{S}]]\right)$, we see that $\hat{A}_{i}^{1}:=(1-\chi(\gamma))^{-1}\left(A_{i} G_{1}^{i}-G_{1}^{i} A_{i}\right)$ and $\hat{A}_{i}^{1} \in$ $M_{n}\left(p^{c+\alpha(k-1)-\mathrm{v}_{\mathrm{p}}(1-\chi(\gamma))} \mathcal{O}_{E}[[\mathcal{S}]]\right)$. We then solve for $\hat{A}_{i}^{2}$ so that $\left(\left(1-\chi(\gamma)^{2}\right)\right) \hat{A}_{i}^{2}$ is an $\mathcal{O}_{E}$-linear combination of products of $A_{i}, \hat{A}_{i}^{1}, G_{1}^{i}, G_{2}^{i}$ which belong to $M_{n}\left(p^{c+\alpha(k-1)-\mathrm{v}_{\mathrm{p}}(1-\chi(\gamma))} \mathcal{O}_{E}[[\mathcal{S}]]\right)$. Dividing this linear combination by $\left(1-\chi(\gamma)^{2}\right)$ we get

$$
\hat{A}_{i}^{2} \in M_{n}\left(p^{c+\alpha(k-1)-\mathrm{v}_{\mathrm{p}}\left((1-\chi(\gamma))\left(1-\chi(\gamma)^{2}\right)\right)} \mathcal{O}_{E}[[\mathcal{S}]]\right) .
$$

Continuing this way we solve for $\hat{A}_{i}^{k-1} \in M_{n}\left(p^{c} \mathcal{O}_{E}[[\mathcal{S}]]\right)$. Part (iv) is clear.

Let $c_{i} \in \mathcal{O}_{E}^{\times}$and let $\Pi(\mathcal{S})=\left(\Pi_{1}, \Pi_{2}, \ldots, \Pi_{f}\right) \in M_{n}^{\mathcal{S}}$ with $\operatorname{det}\left(\Pi_{i}\right)=c_{i} q^{k_{i}}$, where $q=\frac{(1+\pi)^{p}-1}{\pi}$. We denote by $I$ the ideal of $M_{n}\left(\mathcal{O}_{E}[[\mathcal{S}]]\right)$ generated by the set $\left\{p \cdot I d, X_{i} \cdot I d: X_{i} \in \mathcal{S}\right\}$ and by $\overline{M_{n}}$ the quotient ring of $M_{n}\left(\mathcal{O}_{E}[[\mathcal{S}]]\right)$ modulo $I$. We also denote $P_{i}:=\Pi_{i} \bmod I$ for all $i=0,1, \ldots, f-1$. Letting $\varphi$ act trivially on the elements of $\mathcal{S}$, and letting $\varphi(\pi)=(1+\pi)^{p}-1$, we have

$$
\varphi(\vec{\alpha})=\left(\varphi\left(\alpha_{1}\right), \varphi\left(\alpha_{2}\right), \ldots, \varphi\left(\alpha_{0}\right)\right)
$$

for all $\vec{\alpha}=\left(\alpha_{0}, \alpha_{1}, \ldots, \alpha_{f-1}\right) \in \mathcal{O}_{E}[[\pi, \mathcal{S}]]^{|\tau|}$. We denote

$$
\operatorname{Nm}_{\varphi}(\vec{\alpha}):=\vec{\alpha} \cdot \varphi(\vec{\alpha}) \cdots \varphi^{f-1}(\vec{\alpha}),
$$

and for any matrix $A$ in $M_{n}^{\mathcal{S}}$ we write $\operatorname{Nm}_{\varphi}(A):=A \cdot \varphi(A) \cdots \varphi^{f-1}(A)$, with $\varphi$ acting on each entry of the matrix $A$ as in formula (2.3). We fix a matrix $\Pi(\mathcal{S}) \in M_{n}^{\mathcal{S}}$ as above. For the rest of this section we assume that for any $\gamma \in \Gamma_{K}$ there exists a matrix $G_{\gamma}^{(k)}=G_{\gamma}^{(k)}(\mathcal{S}) \in M_{n}^{\mathcal{S}}$ such that:

(a) $G_{\gamma}^{(k)}(\mathcal{S}) \equiv \overrightarrow{I d} \bmod \vec{\pi}$.

(b) $G_{\gamma}^{(k)}(\mathcal{S})-\Pi(\mathcal{S}) \varphi\left(G_{\gamma}^{(k)}(\mathcal{S})\right) \gamma\left(\Pi(\mathcal{S})^{-1}\right) \in \vec{\pi}^{k} M_{n}^{\mathcal{S}}$.

(c) There exist no nonzero matrix $B \in M_{n}\left(\mathcal{O}_{E}[[\mathcal{S}]]\right)^{|\tau|}$ and integer $t>0$ such that $B U=p^{f t} U B$, where $U=\operatorname{Nm}_{\varphi}(\Pi(\mathcal{S}))$.

(d) If $k=k_{i}$ for all $i$, we additionally assume that the operator

$$
\bar{H} \mapsto \overline{H-Q_{f} H\left(p^{f k} Q_{f}^{-1}\right)}: \overline{M_{n}} \rightarrow \overline{M_{n}},
$$

where $Q_{f}=P_{1} P_{2} \cdots P_{f-1} P_{0}$ is surjective. Let $R_{\gamma}^{(k)}(\mathcal{S})$ be the matrices defined by

$$
\vec{\pi}^{k} R_{\gamma}^{(k)}(\mathcal{S}):=G_{\gamma}^{(k)}(\mathcal{S})-\Pi(\mathcal{S}) \varphi\left(G_{\gamma}^{(k)}(\mathcal{S})\right) \gamma\left(\Pi(\mathcal{S})^{-1}\right)
$$

Proposition 2.6. Let $A=\left(A_{1}, A_{2}, \ldots, A_{f}\right) \in M_{n}\left(p^{\alpha(k-1)} \mathcal{O}_{E}\right)^{|\tau|}$ and let $Q_{f}^{A}:=$ $\prod_{i=1}^{f}\left(I d+A_{i}\right) P_{i}$, where $P_{i}=\Pi_{i} \bmod \pi$ for all $i$. Assume that

(1) There exist no nonzero matrix $B \in M_{n}\left(\mathcal{O}_{E}[[\mathcal{S}]]\right)^{|\tau|}$ and integer $t>0$ such that $B U_{A}=p^{f t} U_{A} B$, where $U_{A}=\operatorname{Nm}_{\varphi}((\overrightarrow{I d}+A) P(\mathcal{S}))$, with $P(\mathcal{S})=$ $\Pi(\mathcal{S}) \bmod I$.

If $n=2$, we replace assumption (1) by the following assumption:

$\left(1^{\prime}\right) \operatorname{Tr}\left(Q_{f}^{A}\right) \notin \overline{\mathbb{Q}_{p}}$. 
Let $\hat{A} \in M_{n}\left(\mathcal{O}_{E}[[\pi, \mathcal{S}]]\right)^{|\tau|}$ be as in Lemma 2.5 applied for the matrices $G_{\gamma}(\mathcal{S}):=$ $G_{\gamma}^{(k)}(\mathcal{S})$, where the $G_{\gamma}^{(k)}(\mathcal{S})$ are as in the assumptions preceding this proposition, and let $\Pi_{\hat{A}}(\mathcal{S}):=(\overrightarrow{I d}+\hat{A}) \Pi(\mathcal{S})$. Then for each $\gamma \in \Gamma_{K}$ there exists a unique matrix $G_{\gamma, \hat{A}}(\mathcal{S}) \in M_{n}^{\mathcal{S}}$ such that

(i) $G_{\gamma, \hat{A}}(\mathcal{S}) \equiv \overrightarrow{I d} \bmod \vec{\pi}$ and

(ii) $\Pi_{\hat{A}}(\mathcal{S}) \varphi\left(G_{\gamma, \hat{A}}(\mathcal{S})\right)=G_{\gamma, \hat{A}}(\mathcal{S}) \gamma\left(\Pi_{\hat{A}}(\mathcal{S})\right)$.

Proof. Since $k \geq p$, it follows that $\operatorname{det}\left(I d+A_{i}\right) \in \mathcal{O}_{E}^{\times}$for all $i$. Let $G_{\gamma, \hat{A}}^{(k)}(\mathcal{S}):=$ $G_{\gamma}^{(k)}(\mathcal{S})$ for all $\gamma \in \Gamma_{K}$. The proposition follows from [8, Lemma 4.4] applied for the matrices $\Pi_{\hat{A}}(\mathcal{S})$ and $G_{\gamma, \hat{A}}^{(k)}(\mathcal{S})$ for the case where $\ell=k$. Assumption (1) of this lemma clearly holds. If $k=k_{i}$ for all $i$, since $k \geq p$, the operator

$$
\bar{H} \mapsto \overline{H-Q_{f}^{A} H\left(p^{f k}\left(Q_{f}^{A}\right)^{-1}\right)}: \overline{M_{n}} \rightarrow \overline{M_{n}}
$$

coincides with the operator (2.4) which was assumed to be surjective. Hence assumption (4) of [8, Lemma 4.4] holds. If $n=2$, assumption (3) of [8, Lemma 4.4] holds because of assumption $\left(1^{\prime}\right)$ and [8, Corollary 5.3]. Lemma 2.5](ii) implies that $I d+\hat{A} \in \mathrm{GL}_{n}\left(\mathcal{O}_{E}[[\pi, \mathcal{S}]]\right)^{|\tau|}$. Moreover,

$$
\begin{aligned}
G_{\gamma, \hat{A}}^{(k)}(\mathcal{S})-\Pi_{\hat{A}}(\mathcal{S}) \cdot \varphi\left(G_{\gamma, \hat{A}}^{(k)}(\mathcal{S})\right) \cdot \gamma\left(\Pi_{\hat{A}}(\mathcal{S})^{-1}\right) \\
=G_{\gamma}^{(k)}(\mathcal{S})-(I d+\hat{A}) \cdot \Pi(\mathcal{S}) \cdot \varphi\left(G_{\gamma}^{(k)}(\mathcal{S})\right) \cdot \gamma \Pi(\mathcal{S})^{-1} \cdot \gamma(I d+\hat{A})^{-1} \\
\stackrel{\sqrt{2.5}}{=} G_{\gamma}^{(k)}(\mathcal{S})-(I d+\hat{A}) \cdot G_{\gamma}^{(k)}(\mathcal{S}) \cdot \gamma(I d+\hat{A})^{-1} \\
\quad+\vec{\pi}^{k} \cdot(I d+\hat{A}) \cdot R_{\gamma}^{(k)}(\mathcal{S}) \cdot \gamma(I d+\hat{A})^{-1} .
\end{aligned}
$$

By Lemma $2.5(\mathrm{iii}), G_{\gamma}^{(k)}(\mathcal{S})-(I d+\hat{A}) \cdot G_{\gamma}^{(k)}(\mathcal{S}) \cdot \gamma(I d+\hat{A})^{-1} \in \vec{\pi}^{k} M_{n}^{\mathcal{S}}$, and therefore assumption (2) of [8, Lemma 4.4] holds. This completes the proof.

Proposition 2.7. For any $\vec{a}=\left(a_{0}, a_{1}, \ldots, a_{f-1}\right) \in \mathfrak{m}_{E}^{|\mathcal{S}|}$ and any $\gamma_{1}, \gamma_{2}, \gamma \in \Gamma_{K}$, the following equations hold:

(i) $G_{\gamma_{1} \gamma_{2}, \hat{A}}(\vec{a})=G_{\gamma_{1}, \hat{A}}(\vec{a}) \gamma_{1}\left(G_{\gamma_{2}, \hat{A}}(\vec{a})\right)$ and

(ii) $\Pi_{\hat{A}}(\vec{a}) \varphi\left(G_{\gamma, \hat{A}}(\vec{a})\right)=G_{\gamma, \hat{A}}(\vec{a}) \gamma\left(\Pi_{\hat{A}}(\vec{a})\right)$.

Proof. Both matrices $G_{\gamma_{1} \gamma_{2}, \hat{A}}(\mathcal{S})$ and $G_{\gamma_{1}, \hat{A}}(\mathcal{S}) \gamma_{1}\left(G_{\gamma_{2}, \hat{A}}(\mathcal{S})\right)$ are $\equiv \overrightarrow{I d} \bmod \vec{\pi}$ and are solutions in $B$ of the equation $\Pi(\mathcal{S}) \varphi(B)=B \gamma(\Pi(\mathcal{S}))$. They are equal by the uniqueness part of Proposition 2.6. The second equation follows from conclusion (ii) of the same proposition.

For any $\vec{a} \in \mathfrak{m}_{E}^{|\mathcal{S}|}$ we equip $\mathbb{N}_{\hat{A}}(\vec{a})=\bigoplus_{i=1}^{n}\left(\mathcal{O}_{E}[[\pi]]^{|\tau|}\right) \eta_{i}$ with $\varphi$ and $\Gamma_{K}$-actions defined by $\left(\varphi\left(\eta_{1}\right), \varphi\left(\eta_{2}\right), \ldots, \varphi\left(\eta_{n}\right)\right)=\left(\eta_{1}, \eta_{2}, \ldots, \eta_{n}\right) \Pi_{\hat{A}}(\vec{a})$ and $\left(\gamma \eta_{1}, \gamma \eta_{2}, \ldots\right.$, $\left.\gamma \eta_{n}\right)=\left(\eta_{1}, \eta_{2}, \ldots, \eta_{n}\right) G_{\gamma, \hat{A}}(\vec{a})$ respectively. Proposition 2.7 implies that $\left(\gamma_{1} \gamma_{2}\right) x=$ $\gamma_{1}\left(\gamma_{2} x\right)$ and $\varphi(\gamma x)=\gamma(\varphi(x))$ for all $x \in \mathbb{N}_{\hat{A}}(\vec{a})$ and $\gamma, \gamma_{1}, \gamma_{2} \in \Gamma_{K}$. Since $G_{\gamma, \hat{A}}(\vec{a}) \equiv$ $\overrightarrow{I d} \bmod \vec{\pi}$, it follows that the $\Gamma_{K}$-action on $\mathbb{N}_{\hat{A}}(\vec{a})$ is trivial modulo $\pi \mathbb{N}_{\hat{A}}(\vec{a})$. We have the following. 
Proposition 2.8. For any $\vec{a} \in \mathfrak{m}_{E}^{|\mathcal{S}|}$ the module $\mathbb{N}_{\hat{A}}(\vec{a})$ equipped with the $\varphi$ and $\Gamma_{K}$-actions defined by $\Pi_{\hat{A}}(\vec{a})$ and $G_{\gamma, \hat{A}}(\vec{a})$ respectively is a Wach module corresponding to some $G_{K}$-stable $\mathcal{O}_{E}$-lattice inside some $n$-dimensional crystalline $E$ representation of $G_{K}$ with Hodge-Tate weights in $[-k ; 0]$.

Proof. By Theorem 2.4, the only thing left to prove is that $q^{k} \mathbb{N}_{\hat{A}}(\vec{a}) \subset \varphi^{*}\left(\mathbb{N}_{\hat{A}}(\vec{a})\right)$. This is identical to the proof of [8, Proposition 4.6].

Let $V_{\hat{A}}(\vec{a})=E \otimes_{\mathcal{O}_{E}} \mathrm{~T}_{\hat{A}}(\vec{\alpha})$, where $\mathrm{T}_{\hat{A}}(\vec{\alpha})=\mathbb{T}\left(\mathbb{D}_{\hat{A}}(\vec{a})\right)$ and $\mathbb{D}_{\hat{A}}(\vec{a})=\mathbb{A}_{K, E} \otimes_{\mathbb{A}_{K, E}^{+}}$ $\mathbb{N}_{\hat{A}}(\vec{a})$. By Theorem 2.4 the representations $V_{\hat{A}}(\vec{a})$ are $n$-dimensional crystalline $E$ representations of $G_{K}$ with Hodge-Tate weights in $[-k ; 0]$. They are independent of the lifting $\hat{A}$ of $A$ (see Remark 3.8 below) and instead we denote them by $V_{A}(\vec{a})$. Regarding the $\bmod p$ reductions of these representations, we have the following.

Proposition 2.9. For any $\vec{a} \in \mathfrak{m}_{E}^{|\mathcal{S}|}$ there exist $G_{K}$-stable $\mathcal{O}_{E}$-lattices such that $\bar{V}_{A}(\vec{a}) \simeq \bar{V}_{A}(\overrightarrow{0})$.

Proof. This is identical to the proof of [8, Theorem 4.7], given that $\Pi_{\hat{A}}(\vec{a}) \equiv$ $\Pi_{\hat{A}}(\overrightarrow{0}) \bmod \mathfrak{m}_{E}$ and $G_{\gamma, \hat{A}}(\vec{a}) \equiv G_{\gamma, \hat{A}}(\overrightarrow{0}) \bmod \mathfrak{m}_{E}$.

Lemma 2.10. Let $A \in M_{n}\left(p^{1+\alpha(k-1)} \mathcal{O}_{E}\right)^{|\tau|}$. For any $\gamma \in \Gamma_{K}$ and for $\dagger \in\{0, \hat{A}\}$, let

$G_{\gamma, \dagger}^{(k)}(\mathcal{S})-\Pi_{\dagger}(\mathcal{S}) \varphi\left(G_{\gamma, \dagger}^{(k)}(\mathcal{S})\right) \gamma\left(\Pi_{\dagger}(\mathcal{S})^{-1}\right)=: \vec{\pi}^{k} R_{\gamma, \dagger}^{(k)}$, where $G_{\gamma, \hat{A}}^{(k)}(\mathcal{S}):=G_{\gamma}^{(k)}(\mathcal{S})$.

Then $(i) \Pi_{\hat{A}}(\mathcal{S}) \equiv \Pi(\mathcal{S}) \bmod I$, and $(i i) R_{\gamma, \hat{A}}^{(k)}(\mathcal{S}) \equiv R_{\gamma}^{(k)}(\mathcal{S}) \bmod I$.

Proof. By Lemma 2.5 (iv), $\hat{A} \equiv 0 \bmod p$ and part (i) is clear. The lemma follows immediately by reducing equations (2.7) $\bmod I$.

\section{Proof of the theorem}

Throughout this section we assume that $n=2$. For the rest of the paper we fix an $f$-tuple $P=\left(P_{1}, P_{2}, \ldots, P_{f}\right)$ chosen as in $₫ 1$. For any $A \in M_{2}\left(\mathcal{O}_{E}[[\pi]]\right)^{|\tau|}$ we define $Q_{f}^{A}:=\prod_{i=1}^{i=f}\left(I d+A_{i}\right) P_{i}$ and we let $Q_{f}:=Q_{f}^{0}$. We define

$$
m_{k}:=\left\{\begin{array}{cl}
0 & \text { if } k_{j}=p \text { for all } j \text { and } \operatorname{Tr}\left(Q_{f}\right) \notin \overline{\mathbb{Q}_{p}}, \\
\left\lfloor\frac{k-1}{p-1}\right\rfloor & \text { otherwise. }
\end{array}\right.
$$

For matrices $P$ chosen as in $\oiint 1$ the condition $\operatorname{Tr}\left(Q_{f}\right) \notin \overline{\mathbb{Q}_{p}}$ in the definition of $m_{k}$ turns out to be redundant (see Lemma 3.1 (i) below), and $m_{k}$ coincides with the integer $m$ defined in formula (1.1). Let $\Pi(\mathcal{S})=\left(\Pi_{1}\left(S_{1}\right), \Pi_{2}\left(S_{2}\right), \ldots, \Pi_{f}\left(S_{f}\right)\right) \in$ $M_{n}^{\mathcal{S}}$, where $\Pi_{i}$ are matrices of one of the following four types:

$$
\begin{gathered}
t_{1}:\left(\begin{array}{cc}
c_{i} q^{k_{i}} & 0 \\
S_{i} \varphi\left(z_{i}\right) & 1
\end{array}\right), t_{2}:\left(\begin{array}{cc}
S_{i} \varphi\left(z_{i}\right) & 1 \\
c_{i} q^{k_{i}} & 0
\end{array}\right), \\
t_{3}:\left(\begin{array}{cc}
1 & S_{i} \varphi\left(z_{i}\right) \\
0 & c_{i} q^{k_{i}}
\end{array}\right), t_{4}:\left(\begin{array}{cc}
0 & c_{i} q^{k_{i}} \\
1 & S_{i} \varphi\left(z_{i}\right)
\end{array}\right),
\end{gathered}
$$

with $S_{i} \in \mathcal{S}$ and $c_{i} \in \mathcal{O}_{E}^{\times}$. The $z_{i}$ are polynomials in $\mathbb{Z}_{p}[\pi]$ of degree $\leq k-1$ such that $z_{i} \equiv p^{m_{k}} \bmod \pi$, suitably chosen so that there exist matrices $G_{\gamma}^{(k)}(\mathcal{S}) \in M_{n}^{\mathcal{S}}$ with $G_{\gamma}^{(k)}(\mathcal{S}) \equiv \overrightarrow{I d} \bmod \vec{\pi}$ such that $G_{\gamma}^{(k)}(\mathcal{S})-\Pi(\mathcal{S}) \varphi\left(G_{\gamma}^{(k)}(\mathcal{S})\right) \gamma\left(\Pi(\mathcal{S})^{-1}\right) \in \vec{\pi}^{k} M_{n}^{\mathcal{S}}$. 
The existence of such polynomials has been established in 8 , Proposition 5.9 and Remark 5.12]. We let $\vec{X}=\left(X_{1}, X_{2}, \ldots, X_{f}\right)$ with $\vec{X}=p^{m_{k}} \vec{S}$, and we choose $\Pi$ so that its modulo $\vec{\pi}$ reduction equals $P$. In particular, the type of $\Pi_{i}$ coincides with the type of $P_{i}$ for all $i$. If $A \in M_{2}\left(\mathcal{O}_{E}[[\pi]]\right)^{|\tau|}$, let $\hat{A}$ be a fixed choice of a lifting of $A$ as in Lemma 2.5 with respect to a fixed choice of matrices $G_{\gamma}(\mathcal{S}):=G_{\gamma}^{(k)}(\mathcal{S})$ as above, and let $\Pi_{\hat{A}}(\mathcal{S})=(I d+\hat{A}) \Pi(\mathcal{S})$. Let $E_{i j}, i, j=1,2$, be the $2 \times 2$ matrix with $(i, j)$ entry 1 and all other entries 0.

\section{Lemma 3.1.}

(i) $\operatorname{Tr}\left(Q_{f}\right) \notin \overline{\mathbb{Q}_{p}}$.

(ii) $\operatorname{Tr}\left(Q_{f}\right) \notin p \overline{\mathbb{Z}_{p}}\left[X_{1}, X_{2}, \ldots, X_{f}\right]$.

(iii) For any $A \in M_{2}\left(p \mathcal{O}_{E}[[\pi]]\right)^{|\tau|}, \operatorname{Tr}\left(Q_{f}^{A}\right) \notin \overline{\mathbb{Q}_{p}}$.

(iv) For any $A \in M_{2}\left(p \mathcal{O}_{E}[[\pi]]\right)^{|\tau|}$ the operator (2.6) is surjective.

Proof. For part (i) recall that in the proofs of [8, Theorems 1.5 and 1.7], the types of the coordinate matrices $P_{i}$ of $P$ have been chosen so that $\operatorname{Tr}\left(Q_{f}\right) \notin \overline{\mathbb{Q}_{p}}$. For part (ii), we have

$P_{i} \bmod p=\left\{\begin{array}{l}c\left(k_{i}\right) E_{11}+E_{22}+X_{i} E_{21} \text { if } P_{i}=t_{1}, \\ c\left(k_{i}\right) E_{21}+E_{12}+X_{i} E_{11} \text { if } P_{i}=t_{2}, \\ c\left(k_{i}\right) E_{22}+E_{11}+X_{i} E_{12} \text { if } P_{i}=t_{3}, \\ c\left(k_{i}\right) E_{12}+E_{21}+X_{i} E_{22} \text { if } P_{i}=t_{4},\end{array} \quad\right.$ where $c\left(k_{i}\right)=\left\{\begin{array}{c}0 \text { if } k_{i}>0, \\ 1 \text { if } k_{i}=0 .\end{array}\right.$

The $(i, i)$ entries in $Q_{f} \bmod p$ are sums of distinct terms of the form 1 and $X_{i_{1}}$. $X_{i_{2}} \cdots X_{i_{r_{i}}}$ for some $1 \leq r_{i} \leq f$. Hence $\operatorname{Tr}\left(Q_{f}\right) \not \equiv 0 \bmod p$ (if the diagonal entries of $\operatorname{Tr}\left(Q_{f}\right) \bmod p$ coincide, we use that $p \neq 2$ ). For part (iii), assume that $\operatorname{Tr}\left(Q_{f}^{A}\right) \in \overline{\mathbb{Q}_{p}}$. Since the entries of $Q_{f}^{A}$ are in $\overline{\mathbb{Z}_{p}}\left[X_{1}, X_{2}, \ldots, X_{f}\right]$ it follows that $\operatorname{Tr}\left(Q_{f}^{A}\right) \in \overline{\mathbb{Z}_{p}}$. Since $Q_{f}^{A} \equiv Q_{f} \bmod p$ it follows that $\operatorname{Tr}\left(Q_{f}^{A}\right) \equiv \operatorname{Tr}\left(Q_{f}\right) \bmod$ $p$ and therefore that $\operatorname{Tr}\left(Q_{f}\right) \in \overline{\mathbb{Z}_{p}}+p \overline{\mathbb{Z}_{p}}\left[X_{1}, X_{2}, \ldots, X_{f}\right]$. Since $\operatorname{Tr}\left(Q_{f}\right) \notin \overline{\mathbb{Q}_{p}}$, Lemma 5.19 and Corollary 5.17 of [8] imply that $Q_{f} \bmod I=E_{12}$ or $E_{21}$; therefore $\operatorname{Tr}\left(Q_{f}\right) \equiv 0 \bmod \left(p, X_{1}, \ldots, X_{f}\right)$. Hence $\operatorname{Tr}\left(Q_{f}\right) \in\left(\overline{\mathbb{Z}_{p}}+p \overline{\mathbb{Z}_{p}}\left[X_{1}, X_{2}, \ldots, X_{f}\right]\right) \cap$ $\left(p, X_{1}, \ldots, X_{f}\right)=p \overline{\mathbb{Z}_{p}}\left[X_{1}, X_{2}, \ldots, X_{f}\right]$, which contradicts part (ii) of the lemma. Part (iv) for $A=0$ follows from [8, Corollary 5.20]. The general case holds because the operators with any $A \in M_{2}\left(p \mathcal{O}_{E}[[\pi]]\right)^{|\tau|}$ coincide with those with $A=0$.

Proposition 3.2. Let $A \in M_{2}\left(p^{\alpha(k-1)} \mathcal{O}_{E}[[\pi]]\right)^{|\tau|}$ and let $\Pi_{\hat{A}}(\mathcal{S})$ be as in the beginning of $₫ 3$. For each $\gamma \in \Gamma_{K}$ there exists a unique matrix $G_{\gamma}(\mathcal{S}) \in M_{2}^{\mathcal{S}}$ such that

(i) $G_{\gamma}(\mathcal{S}) \equiv \overrightarrow{I d} \bmod \vec{\pi}$;

(ii) $\Pi_{\hat{A}}(\mathcal{S}) \varphi\left(G_{\gamma}(\mathcal{S})\right)=G_{\gamma}(\mathcal{S}) \gamma \Pi_{\hat{A}}(\mathcal{S})$ for all $\gamma$.

Proof. Conditions (a) and (b) preceding Proposition 2.6 hold by the discussion in the beginning of 93 . Condition (c) preceding Proposition 2.6 and Condition (1') of Proposition 2.6 hold because $\operatorname{Tr}\left(Q_{f}\right) \notin \overline{\mathbb{Q}_{p}}$ and $\operatorname{Tr}\left(Q_{f}^{A}\right) \notin \overline{\mathbb{Q}_{p}}$ respectively by Lemma 3.1 (i) and (iii). Finally, Condition (d) preceding Proposition 2.6 holds by Lemma 3.1 (iv) with $A=0$. The proposition follows by Proposition 2.6. 
For any $\vec{a} \in \mathfrak{m}_{E}^{|\mathcal{S}|}$ and $\dagger \in\{0, \hat{A}\}$, we equip $\mathbb{N}_{\dagger}(\vec{a})=\left(\mathcal{O}_{E}[[\pi]]^{|\tau|}\right) \eta_{1} \oplus\left(\mathcal{O}_{E}[[\pi]]^{|\tau|}\right) \eta_{2}$ with the $\varphi$ and $\Gamma_{K}$-actions defined defined by $\left(\varphi\left(\eta_{1}\right), \varphi\left(\eta_{2}\right)\right)=\left(\eta_{1}, \eta_{2}\right) \Pi_{\dagger}(\vec{a})$ and $\left(\gamma \eta_{1}, \gamma \eta_{2}\right)=\left(\eta_{1}, \eta_{2}\right) G_{\gamma, \dagger}(\vec{a})$ respectively.

Corollary 3.3. The module $\mathbb{N}_{\dagger}(\vec{a})$ with the above $\varphi$ and $\Gamma_{K}$-actions is a Wach module corresponding to some $G_{K}$-stable $\mathcal{O}_{E}$-lattice of a 2-dimensional crystalline E-representation $V_{\dagger}(\vec{a})$ of $G_{K}$ with Hodge-Tate weights in $[-k ; 0]$.

Proof. Follows immediately from Proposition 2.8

As in $\$ 2.2$, the representation $V_{\hat{A}}(\vec{a})$ is independent of the lifting $\hat{A}$ and we simply write $V_{A}(\vec{a})$.

Lemma 3.4. If $s \geq k+1$ and $B \in M_{2}\left(\mathcal{O}_{E}[[\mathcal{S}]]\right)^{|\tau|}$ is such that

$$
B \equiv Q_{f} B\left(p^{f(s-1)} Q_{f}^{-1}\right) \bmod I,
$$

then $B \equiv 0 \bmod I$.

Proof. We may assume that $s-1=k=k_{i}$ for all $i$; otherwise $Q_{f} B\left(p^{f(s-1)} Q_{f}^{-1}\right) \equiv$ 0 mod $I$ and the lemma holds trivially. By Lemma 3.1 (i), $\operatorname{Tr}\left(Q_{f}\right) \notin \overline{\mathbb{Q}_{p}}$ and [8. Lemma 5.19 and Corollary 5.17] (where in [8] $\overline{Q_{f}}:=Q_{f} \bmod I$ ) imply that $Q_{f} \bmod I=E_{12}$ or $E_{21}$. Recall that $k \geq p$.

Claim. If $Q_{f} \bmod I=E_{i j}$ with $i \neq j$, then $p^{f k} Q_{f}^{-1} \bmod I=-Q_{f} \bmod I$. If $Q_{f} \bmod I=E_{11}$, then $p^{f k} Q_{f}^{-1} \bmod I=E_{22}$, and if $Q_{f} \bmod I=E_{22}$, then $p^{f k} Q_{f}^{-1} \bmod I=E_{11}$.

Proof of the Claim. By induction on $f$. For $f=1$, formula (3.1) becomes

$$
P \bmod I= \begin{cases}E_{22} & \text { if } P=t_{1}, \\ E_{12} & \text { if } P=t_{2}, \\ E_{11} & \text { if } P=t_{3}, \\ E_{21} & \text { if } P=t_{4},\end{cases}
$$

and the claim is clear. Suppose $f \geq 2$. Case (i). $Q_{f} \bmod I=E_{12}$. If $P_{1} P_{2} \cdots P_{f-1}$ $\bmod I=E_{11}$, then $P_{f} \bmod I=E_{12}$. The matrix $P_{f}$ is of type 2 , and by the inductive hypothesis

$p^{k f} Q_{f}^{-1} \bmod I=\left(p^{k} P_{f}^{-1}\right) \cdot\left(\left(p^{k} P_{f-1}^{-1}\right) \cdots\left(p^{k} P_{1}^{-1}\right)\right) \bmod I=-E_{12} \cdot E_{22}=-E_{12}$. If $P_{1} P_{2} \cdots P_{f-1} \bmod I=E_{12}$, then $P_{f} \bmod I=E_{22}$. The matrix $P_{f}$ is of type 1 , and by the inductive hypothesis

$p^{k f} Q_{f}^{-1} \bmod I=\left(p^{k} P_{f}^{-1}\right) \cdot\left(\left(p^{k} P_{f-1}^{-1}\right) \cdots\left(p^{k} P_{1}^{-1}\right)\right) \bmod I=E_{11} \cdot\left(-E_{12}\right)=-E_{12}$.

The Claim follows by the inductive hypothesis, arguing similarly for the other possibilities for $Q_{f} \bmod I$.

The Claim combined with the formula $B \equiv Q_{f} B\left(p^{f k} Q_{f}^{-1}\right) \bmod I$ implies that $B \equiv-E_{i j} B E_{i j} \bmod I$ with $i \neq j$. From the latter it is immediate that $B \equiv 0 \bmod I$.

Proposition 3.5. Let $A \in M_{2}\left(p^{1+\alpha(k-1)} \mathcal{O}_{E}[[\pi]]\right)^{|\tau|}$ and let $\Pi_{\hat{A}}(\mathcal{S})$ and $G_{\gamma, \hat{A}}(\mathcal{S})$ be as in Proposition 2.6. Then $\Pi_{\hat{A}}(\mathcal{S}) \equiv \Pi(\mathcal{S}) \bmod I$ and $G_{\gamma, \hat{A}}(\mathcal{S}) \equiv G_{\gamma}(\mathcal{S}) \bmod I$. 
Proof. By Lemma 2.5 (iv), $\hat{A} \equiv 0 \bmod I$; hence $\Pi_{\hat{A}}(\mathcal{S}) \equiv \Pi(\mathcal{S}) \bmod I$. Fix a topological generator $\gamma$ of $\Gamma_{K}$. By the proofs of [8, Propositions 5.9 and 5.11], there exist a matrix $G_{\gamma}^{(k)}(\mathcal{S}) \in M_{n}^{\mathcal{S}}$ with $G_{\gamma}^{(k)}(\mathcal{S}) \equiv \overrightarrow{I d} \bmod \vec{\pi}$ and a matrix $R^{(k)}(\mathcal{S}) \in M_{n}^{\mathcal{S}}$ such that

$$
G_{\gamma}^{(k)}(\mathcal{S})-\Pi(\mathcal{S}) \cdot \varphi\left(G_{\gamma}^{(k)}(\mathcal{S})\right) \cdot \gamma\left(\Pi(\mathcal{S})^{-1}\right)=\vec{\pi}^{k} R^{(k)}(\mathcal{S})
$$

Moreover, by the proof of [8, Lemma 4.1], for all $s \geq k+1$ there exist matrices $G_{\gamma}^{(s)}(\mathcal{S}) \in M_{n}^{\mathcal{S}}$ and $R^{(s)}(\mathcal{S}) \in M_{n}^{\mathcal{S}}$ such that $G_{\gamma}^{(s)}(\mathcal{S}) \equiv G_{\gamma}^{(s-1)}(\mathcal{S}) \bmod \vec{\pi}^{s-1} M_{n}^{\mathcal{S}}$ and

$$
G_{\gamma}^{(s)}(\mathcal{S})-\Pi(\mathcal{S}) \cdot \varphi\left(G_{\gamma}^{(s)}(\mathcal{S})\right) \cdot \gamma\left(\Pi(\mathcal{S})^{-1}\right)=\vec{\pi}^{s} R^{(s)}(\mathcal{S}) .
$$

Arguing as in the proof of part (ii) of Proposition 2.6 and taking into account equations (3.2) and (3.3), we see that for all $s \geq k$ there exist matrices $R_{\hat{A}}^{(s)}(\mathcal{S}) \in$ $M_{n}^{\mathcal{S}}$ such that

$$
G_{\gamma, \hat{A}}^{(s)}(\mathcal{S})-\Pi_{\hat{A}}(\mathcal{S}) \cdot \varphi\left(G_{\gamma, \hat{A}}^{(s)}(\mathcal{S})\right) \cdot \gamma\left(\Pi_{\hat{A}}(\mathcal{S})^{-1}\right)=\vec{\pi}^{s} R_{\hat{A}}^{(s)}(\mathcal{S}) .
$$

Combining equations (3.2), (3.3) and (3.4) for all $s \geq k$ we write

$$
G_{\gamma, \dagger}^{(s)}(\mathcal{S})-\Pi_{\dagger}(\mathcal{S}) \cdot \varphi\left(G_{\gamma, \dagger}^{(s)}(\mathcal{S})\right) \cdot \gamma\left(\Pi_{\dagger}(\mathcal{S})^{-1}\right)=\vec{\pi}^{s} R_{\dagger}^{(s)}(\mathcal{S}),
$$

with $\dagger \in\{0, \hat{A}\}$. We defined $G_{\gamma, \hat{A}}^{(k)}(\mathcal{S}):=G_{\gamma}^{(k)}(\mathcal{S})$, and by Lemma2.10 (ii), $R_{\gamma, \hat{A}}^{(k)}(\mathcal{S}) \equiv$ $R_{\gamma}^{(k)}(\mathcal{S}) \bmod I$. We will show by induction that $G_{\gamma, \hat{A}}^{(s)}(\mathcal{S}) \equiv G_{\gamma}^{(s)}(\mathcal{S}) \bmod I$ and $R_{\hat{A}}^{(s)}(\mathcal{S}) \equiv R^{(s)}(\mathcal{S}) \bmod I$ for all $s \geq k$. For $s \geq k+1$, let $G_{\gamma, \dagger}^{(s)}=G_{\gamma, \dagger}^{(s-1)}+\vec{\pi}^{s-1} H_{\dagger}^{(s)}$, where $H_{\dagger}^{(s)}=H_{\gamma, \dagger}^{(s)} \in M_{n}\left(\mathcal{O}_{E}[[\mathcal{S}]]\right)^{|\tau|}$, and let $R_{\dagger}^{(s)}(\mathcal{S})=\bar{R}_{\dagger}^{(s)}(\mathcal{S})+\vec{\pi} \cdot C_{\dagger}^{(s)}$ for some matrices $\bar{R}_{\dagger}^{(s)}(\mathcal{S}) \in M_{n}\left(\mathcal{O}_{E}[[\mathcal{S}]]\right)^{|\tau|}$ and $C_{\dagger}^{(s)} \in M_{n}^{\mathcal{S}}$. By the inductive hypothesis, $\bar{R}_{\hat{A}}^{(s-1)}(\mathcal{S})+\vec{\pi} \cdot C_{\hat{A}}^{(s-1)} \equiv \bar{R}^{(s-1)}(\mathcal{S})+\vec{\pi} \cdot C^{(s-1)} \bmod I$, and since $\bar{R}_{\dagger}^{(s-1)}(\mathcal{S}) \in$ $M_{n}\left(\mathcal{O}_{E}[[\mathcal{S}]]\right)^{|\tau|}$, the latter implies that $\bar{R}_{\hat{A}}^{(s-1)}(\mathcal{S}) \equiv \bar{R}^{(s-1)}(\mathcal{S}) \bmod I$. Let $\Pi_{\dagger}(\mathcal{S})=$ $\Pi_{\dagger}^{(0)}+\pi \Pi_{\dagger}^{(1)}+\pi^{2} \Pi_{\dagger}^{(2)}+\cdots$, and let $\Pi_{\dagger}^{(0)}=\left(P_{1, \dagger}, P_{2, \dagger}, \ldots, P_{f-1, \dagger}, P_{0, \dagger}\right)$. By the proof of [8, Lemma 4.1], the matrices $H_{\dagger}^{(s)}$ can be chosen to be solutions of the equation

$$
H_{\dagger}^{(s)}-\vec{p}^{(s-1)} \Pi_{\dagger}^{(0)}(\mathcal{S}) \varphi\left(H_{\dagger}^{(s)}\right)\left(\Pi_{\dagger}^{(0)}(\mathcal{S})\right)^{-1}=-\bar{R}_{\dagger}^{(s-1)}, \text { with } \dagger \in\{0, \hat{A}\} .
$$

Let $H_{\dagger}^{(s)}=\left(H_{1, \uparrow}^{(s)}, H_{2, \uparrow}^{(s)}, \ldots, H_{f-1, \uparrow}^{(s)}, H_{0, \uparrow}^{(s)}\right)$ and

$$
-\bar{R}_{\dagger}^{(s-1)}=\left(\bar{R}_{1, \dagger}^{(s-1)}, \bar{R}_{2, \dagger}^{(s-1)}, \ldots, \bar{R}_{f-1, \dagger}^{(s-1)}, \bar{R}_{0, \dagger}^{(s-1)}\right) .
$$

Equation (3.6) is equivalent to the system of equations

$$
H_{i, \dagger}^{(s)}-P_{i, \dagger} \cdot H_{i+1, \dagger}^{(s)} \cdot\left(p^{s-1} P_{i, \dagger}^{-1}\right)=\bar{R}_{i, \dagger}^{(s-1)},
$$

for $i=1,2, \ldots, f, \dagger \in\{0, \hat{A}\}$, and with indices viewed $\bmod f$. These imply that

$$
\begin{aligned}
H_{1, \dagger}^{(s)}-Q_{f, \dagger} H_{1, \dagger}^{(s)}\left(p^{f(s-1)} Q_{f, \dagger}^{-1}\right)= & \bar{R}_{1, \dagger}^{(s-1)}+Q_{1, \dagger} \bar{R}_{2, \dagger}^{(s-1)}\left(p^{(s-1)} Q_{1, \dagger}^{-1}\right) \\
& +Q_{2, \dagger} \bar{R}_{3, \dagger}^{(s-1)}\left(p^{2(s-1)} Q_{2, \dagger}^{-1}\right) \\
& +\cdots+Q_{f-1, \dagger} \bar{R}_{0, \dagger}^{(s-1)}\left(p^{(s-1)(f-1)} Q_{f-1, \dagger}^{-1}\right),
\end{aligned}
$$


where $Q_{i, \uparrow}=P_{1, \uparrow} \cdots P_{i, \uparrow}$ for all $i=1,2, \ldots, f$. The matrices $H_{i, \uparrow}^{(s)}, i=2,3, \ldots, f$ are uniquely determined by the matrix $H_{1, \dagger}^{(s)}$. Let $V_{\dagger}^{(s)}=\bar{R}_{1, \dagger}^{(s-1)}+Q_{1, \dagger} \bar{R}_{2, \dagger}^{(s-1)}\left(p^{(s-1)} Q_{1, \dagger}^{-1}\right)$ $+Q_{2, \dagger} \bar{R}_{3, \dagger}^{(s-1)}\left(p^{2(s-1)} Q_{2, \dagger}^{-1}\right)+\cdots+Q_{f-1, \dagger} \bar{R}_{0, \dagger}^{(s-1)}\left(p^{(s-1)(f-1)} Q_{f-1, \dagger}^{-1}\right)$. Since $\bar{R}_{i}^{(s-1)} \equiv$ $\bar{R}_{i, \hat{A}}^{(s-1)} \bmod I$, and since $Q_{i} \equiv Q_{i, \hat{A}} \bmod I$ and $p^{i(s-1)} Q_{i, \hat{A}}^{-1} \equiv p^{i(s-1)} Q_{i}^{-1} \bmod I$ for all $i$, it follows that $V^{(s)} \equiv V_{\hat{A}}^{(s)} \bmod I$. Since $Q_{1, \hat{A}} \equiv Q_{1} \bmod I$, the latter and equation (3.8) imply that $H_{1, \hat{A}}^{(s)}-H_{1}^{(s)}=Q_{f}\left(H_{1, \hat{A}}^{(s)}-H_{1}^{(s)}\right)\left(p^{f(s-1)} Q_{f}^{-1}\right) \bmod I$, and Lemma 3.4 applied for $B=H_{1, \hat{A}}^{(s)}-H_{1}^{(s)}$ implies that $H_{1, \hat{A}}^{(s)} \equiv H_{1}^{(s)} \bmod I$. Since $P_{i, \hat{A}} \equiv P_{i} \bmod I$ and $R_{i, \hat{A}}^{(s-1)} \equiv R_{i}^{(s-1)} \bmod I$, equation (3.7) implies that $H_{\hat{A}}^{(s)} \equiv H^{(s)} \bmod I$. Since $G_{\gamma, \dagger}^{(s)}=G_{\gamma, \dagger}^{(s-1)}+\vec{\pi}^{s-1} H_{\dagger}^{(s)}$, the inductive hypothesis implies that $G_{\gamma, \hat{A}}^{(s)}(\mathcal{S}) \equiv G_{\gamma}^{(s)}(\mathcal{S}) \bmod I$. Formula (3.5) yields $\vec{\pi}^{s} \cdot \gamma \Pi_{\hat{A}}(\mathcal{S})$. $R_{\hat{A}}^{(s)} \equiv \vec{\pi}^{s} \cdot \gamma \Pi(\mathcal{S}) \cdot R^{(s)} \bmod I$. Since the coordinate matrices of both the matrices $\gamma \Pi_{\hat{A}}(\mathcal{S})$ and $\gamma \Pi(\mathcal{S})$ coincide $\bmod I$ and have non-zero determinants mod $I$, it follows that $R_{\hat{A}}^{(s)} \equiv R^{(s)} \bmod I$, and this finishes the induction. We have shown that $G_{\gamma, \hat{A}}^{(s)}(\mathcal{S}) \equiv G_{\gamma}^{(s)}(\mathcal{S}) \bmod I$ for any $s \geq k$. Since $G_{\gamma, \dagger}(\mathcal{S})=\lim _{s \rightarrow \infty} G_{\gamma, \dagger}^{(s)}(\mathcal{S})$ it follows that $G_{\gamma, \hat{A}}(\mathcal{S}) \equiv G_{\gamma}(\mathcal{S}) \bmod I$, and this finishes the proof.

Corollary 3.6. Let $A \in M_{2}\left(p^{1+\alpha(k-1)} \mathcal{O}_{E}[[\pi]]\right)^{|\tau|}$. Then for any $\vec{a} \in \mathfrak{m}_{E}^{f}$ there exist $G_{K_{f}}$-stable $\mathcal{O}_{E}$-lattices with respect to which $\bar{V}_{A}(\vec{a}) \simeq \bar{V}_{0}(\overrightarrow{0})$.

Proof. Proposition 3.5 implies that $\Pi_{\hat{A}}(\vec{a}) \equiv \Pi(\overrightarrow{0}) \bmod \mathfrak{m}_{E}$ and

$$
G_{\gamma, \hat{A}}(\vec{a}) \equiv G_{\gamma}(\overrightarrow{0}) \bmod \mathfrak{m}_{E}
$$

for all $\gamma \in \Gamma_{K}$. The rest of the proof is identical to that of [8, Theorem 4.7].

Parts (ii) and (iii) of Theorem A follow from Proposition 2.9 and Corollary 3.6. The following proposition proves part (i) and finishes the proof of the theorem.

Proposition 3.7. Let $A \in M_{2}\left(p^{\alpha(k-1)} \mathcal{O}_{E}[[\pi]]\right)^{|\tau|}$ and $\vec{\alpha} \in\left(p^{m} \mathfrak{m}_{E}\right)^{f}$. We define the rank two filtered $\varphi$-module $\left(\mathbb{D}_{A}(\vec{\alpha}), \varphi\right)$ with Frobenius endomorphism

$$
\left(\varphi\left(\eta_{1}\right), \varphi\left(\eta_{2}\right)\right):=\left(\eta_{1}, \eta_{2}\right) P_{A}(\vec{\alpha})
$$

and filtration

$$
\operatorname{Fil}^{\mathrm{j}}\left(\mathbb{D}_{A}(\vec{\alpha})\right):=\left\{\begin{array}{cl}
E^{|\tau|} \eta_{1} \oplus E^{|\tau|} \eta_{2} & \text { if } j \leq 0, \\
E^{\left|\tau_{I_{0}}\right|}\left(\vec{x} \eta_{1}+\vec{y} \eta_{2}\right) & \text { if } 1 \leq j \leq w_{0}, \\
E^{\left|\tau_{I_{1}}\right|}\left(\vec{x} \eta_{1}+\vec{y} \eta_{2}\right) & \text { if } 1+w_{0} \leq j \leq w_{1}, \\
\ldots & \ldots \\
E^{\left|\tau_{I_{t-1}}\right|}\left(\vec{x} \eta_{1}+\vec{y} \eta_{2}\right) & \text { if } 1+w_{t-2} \leq j \leq w_{t-1}, \\
0 & \text { if } j \geq 1+w_{t-1},
\end{array}\right.
$$

with

$$
\left(x_{i}, y_{i}\right)= \begin{cases}\left(1,-\alpha_{i}\right) & \text { if } P_{i} \text { has type } 1 \text { or } 2 \\ \left(-\alpha_{i}, 1\right) & \text { if } P_{i} \text { has type } 3 \text { or } 4\end{cases}
$$


The filtered $\varphi$-modules $\mathbb{D}_{A}(\vec{\alpha})$ are admissible and correspond to 2-dimensional crystalline E-linear representations $V_{A}(\vec{\alpha})$ of $G_{K_{f}}$ with Hodge-Tate type $\operatorname{HT}\left(\tau_{i}\right)=$ $\left\{0,-k_{i}\right\}$.

Proof. We compute the filtered $\varphi$-modules given rise to by the Wach modules $\mathbb{N}_{\hat{A}}(\vec{a})$. The $\varphi$-action is obviously given by formula (3.9), and it suffices to compute Fil ${ }^{\mathrm{j}}\left(\mathbb{N}_{\hat{A}}(\vec{a}) / \pi \mathbb{N}_{\hat{A}}(\vec{a})\right)$. By Theorem $2.4, \vec{x} \eta_{1}+\vec{y} \eta_{2} \in \mathrm{Fil}^{\mathrm{j}}\left(\mathbb{N}_{\hat{A}}(\vec{a})\right)$ if and only if $\varphi\left(\vec{x} \eta_{1}+\vec{y} \eta_{2}\right) \in q^{j} \mathbb{N}_{\hat{A}}(\vec{a})$. Written in matrix form and recalling that the $\varphi$-action on $\mathcal{O}_{E}[[\pi]]^{|\tau|}$ is given by formula (2.1), the latter is equivalent to the existence of vectors $\vec{u}_{1}, \vec{u}_{2} \in \mathcal{O}_{E}[[\pi]]^{|\tau|}$ such that

$$
\begin{gathered}
\left(e_{i-1} \eta_{1}, e_{i-1} \eta_{2}\right)\left(I d+\hat{A}_{i}(\vec{a})\right) \Pi_{i}\left(a_{i}\right)\left(\varphi\left(x_{i}\right), \varphi\left(y_{i}\right)\right)^{t} \\
=\left(q^{j} e_{i-1} \eta_{1}, q^{j} e_{i-1} \eta_{2}\right)\left(u_{1}^{i-1}, u_{2}^{i-1}\right)^{t}
\end{gathered}
$$

for all $i=0,1, \ldots, f-1$, where $e_{i}$ are the idempotents of $\mathcal{O}_{E}[[\pi]]^{|\tau|}$. Let

$$
\left(\zeta_{1}^{i-1}, \zeta_{2}^{i-1}\right):=\left(e_{i-1} \eta_{1}, e_{i-1} \eta_{2}\right)\left(I d+\hat{A}_{i}(\vec{a})\right)
$$

and

$$
\left(v_{1}^{i-1}, v_{2}^{i-1}\right)^{t}:=\left(I d+\hat{A}_{i}(\vec{a})\right)^{-1}\left(u_{1}^{i-1}, u_{2}^{i-1}\right)^{t} .
$$

Since $\hat{A} \equiv A \bmod \vec{\pi}$ and $\operatorname{det}\left(I d+A_{i}\right) \in \mathcal{O}_{E}^{\times}$it follows that $I d+\hat{A}_{i}(\vec{a}) \in \mathrm{GL}_{2}\left(\mathcal{O}_{E}[[\pi]]\right)$ for all $i$. Therefore $\left(\zeta_{1}^{i}, \zeta_{2}^{i}\right)$ is an ordered basis of $e_{i} \mathbb{N}_{\hat{A}}(\vec{a})$ for all $i$ and equation (3.11) implies

$$
\left(\zeta_{1}^{i-1}, \zeta_{2}^{i-1}\right) \Pi_{i}\left(a_{i}\right)\left(\varphi\left(x_{i}\right), \varphi\left(y_{i}\right)\right)^{t}=\left(q^{j} \zeta_{1}^{i-1}, q^{j} \zeta_{2}^{i-1}\right)\left(v_{1}^{i-1}, v_{2}^{i-1}\right)^{t} .
$$

Assume that $\Pi_{i}\left(X_{i}\right)$ is of type 2. Arguing as in the proof of [8, Proposition 5.22] we see that $x_{i}, y_{i} \equiv 0 \bmod \pi$ if $j \geq k_{i}$ and $\pi^{j} \mid x_{i}+y_{i} a_{i} z_{i}$ for $1 \leq j \leq k_{i}$. Since $z_{i} \bmod \pi=p^{m}$ and $\alpha_{i}:=p^{m} a_{i}$,

$$
e_{i} \vec{x} \eta_{1}+e_{i} \vec{y} \eta_{2}+\pi \mathbb{N}_{\hat{A}}(\vec{a})=\left\{\begin{array}{cl}
\alpha_{i} \bar{y}_{i} e_{i} \eta_{1}+\bar{y}_{i} e_{i} \eta_{2}+\pi \mathbb{N}_{\hat{A}}(\vec{a}) & \text { if } 1 \leq j \leq k_{i}, \\
0 & \text { if } j \geq k_{i},
\end{array}\right.
$$

where $\bar{y}_{i}=y_{i} \bmod \pi$ can be any element of $\mathcal{O}_{E}$. Hence

$$
e_{i} \operatorname{Fil}^{\mathrm{j}}\left(\mathbb{N}_{\hat{A}}(\vec{a}) / \pi \mathbb{N}_{\hat{A}}(\vec{a})\right)=\left\{\begin{array}{cl}
e_{i}\left(\mathcal{O}_{E}^{|\tau|}\right) \eta_{1} \oplus e_{i}\left(\mathcal{O}_{E}^{|\tau|}\right) \eta_{2} & \text { if } j \leq 0, \\
e_{i}\left(\mathcal{O}_{E}^{|\tau|}\right)\left(\vec{x} \eta_{1}+\vec{y} \eta_{2}\right) & \text { if } 1 \leq j \leq k_{i}, \\
0 & \text { if } j \geq 1+k_{i},
\end{array}\right.
$$

with $\left(x_{i}, y_{i}\right)=\left(-\alpha_{i}, 1\right)$. Computing for the other choices of $\Pi_{i}\left(a_{i}\right)$, we see that for all $i \in I_{0},\left(x_{i}, y_{i}\right)$ is as in formula (3.10) and the proof follows as in [8, Proposition 5.22]. To finish the proof, notice that by the definition of the polynomials $z_{i}$ appearing in the matrices $\Pi_{i}$, the sets $\left\{\left(z_{1} a_{1} \bmod \pi, \ldots, z_{0} a_{0} \bmod \pi\right)\right\}$, where $\left(a_{1}, \ldots, a_{f}\right) \in \mathfrak{m}_{E}^{f}$, and $\left(p^{m} \mathfrak{m}_{E}\right)^{f}$ coincide. We let $\vec{\alpha}:=p^{m} \cdot \vec{a}$ for any vector $\vec{a} \in \mathfrak{m}_{E}^{f}$ and parametrize our families by the vectors $\vec{\alpha}$. Finally, since $\mathbb{D}_{A}(\vec{\alpha})=\operatorname{Fil}^{\mathrm{j}} \mathbb{D}_{\text {cris }}\left(V_{A}(\vec{\alpha})\right) \cong E^{|\tau|} \otimes_{\mathcal{O}_{E}|\tau|}$ $\operatorname{Fil}^{\mathrm{j}}\left(\mathbb{N}_{\hat{A}}(\vec{a}) / \pi \mathbb{N}_{\hat{A}}(\vec{a})\right)$, the filtered $\varphi$-modules $\mathbb{D}_{A}(\vec{\alpha})$ are admissible because the $E^{|\tau|} \otimes_{\mathcal{O}_{E}|\tau|}\left(\mathbb{N}_{\hat{A}}(\vec{a}) / \pi \mathbb{N}_{\hat{A}}(\vec{a})\right)$ are admissible by Theorem 2.4 .

Remark 3.8. For fixed $\vec{a}$ the filtered $\varphi$-modules $\mathbb{N}_{\hat{A}}(\vec{a}) / \pi \mathbb{N}_{\hat{A}}(\vec{a})$ depend only on $A \equiv \hat{A} \bmod \pi$. This is clear from the proof of Proposition 3.7 (essentially by Theorem 2.4). 


\section{ACKNOWLEDGEMENTS}

The paper was written at the Fields Institute during the Thematic Program on Galois Representations in spring 2012. The author thanks the Fields Institute for its hospitality and financial support, and the anonymous referee for a very careful reading of the paper.

\section{REFERENCES}

[1] Laurent Berger, Limites de représentations cristallines (French, with English and French summaries), Compos. Math. 140 (2004), no. 6, 1473-1498. MR2098398 (2006c:11138)

[2] L. Berger, Local constancy for the reduction mod $p$ of 2-dimensional crystalline representations, Bull. Lond. Math. Soc. 44 (2012), no. 3, 451-459. MR2966990

[3] L. Berger and C. Breuil, Towards a p-adic Langlands programme. Summer school on p-adic arithmetic geometry in Hangzhou. http://perso.ens-lyon.fr/laurent.berger/ autrestextes/hangzhou.pdf

[4] Laurent Berger, Hanfeng Li, and Hui June Zhu, Construction of some families of 2dimensional crystalline representations, Math. Ann. 329 (2004), no. 2, 365-377, DOI 10.1007/s00208-004-0529-y. MR2060368(2005k:11104)

[5] Kevin Buzzard, Fred Diamond, and Frazer Jarvis, On Serre's conjecture for mod $\ell$ Galois representations over totally real fields, Duke Math. J. 155 (2010), no. 1, 105-161, DOI 10.1215/00127094-2010-052. MR2730374 (2012k:11067)

[6] Pierre Colmez and Jean-Marc Fontaine, Construction des représentations p-adiques semistables (French), Invent. Math. 140 (2000), no. 1, 1-43, DOI 10.1007/s002220000042. MR.1779803 (2001g:11184)

[7] Gerasimos Dousmanis, Rank two filtered $(\phi, N)$-modules with Galois descent data and coefficients, Trans. Amer. Math. Soc. 362 (2010), no. 7, 3883-3910, DOI 10.1090/S0002-9947-1005100-7. MR2601613 (2011e:11099)

[8] Gerasimos Dousmanis, On reductions of families of crystalline Galois representations, Doc. Math. 15 (2010), 873-938. MR2745686 (2012g:11104)

[9] J.-M. Fontaine, Le corpes des périodes p-adiques. With an appendix by Pierre Colmez. Périodes $p$-adiques (Bures-sur-Yvette, 1988), Astérisque 223 (1994), 59-111. MR.1293971 (95k:11086)

[10] Jean-Marc Fontaine, Représentations p-adiques des corps locaux. I (French), The Grothendieck Festschrift, Vol. II, Progr. Math., vol. 87, Birkhäuser Boston, Boston, MA, 1990, pp. 249-309. MR.1106901 (92i:11125)

[11] T. Gee, T. Liu, and D. Savitt, The Buzzard-Diamond-Jarvis conjecture for unitary groups, J. Amer. Math. Soc. 27 (2014), no. 2, 389-435. MR.3164985

[12] Tong Liu, A note on lattices in semi-stable representations, Math. Ann. 346 (2010), no. 1, 117-138, DOI 10.1007/s00208-009-0392-y. MR2558890(2011d:11272)

Fields Institute for Mathematics, 222 College Street, Toronto, Ontario, M5T 3J1 CANADA

Current address: Athens, Greece

E-mail address: makis.dousmanis@gmail.com 